\title{
Multi-frequency imaging of the galaxy cluster Abell 2163 using the Sunyaev-Zel'dovich effect
}

\author{
M. Nord ${ }^{1,2}$, K. Basu ${ }^{1,2}$, F. Pacaud ${ }^{1}$, P. A. R. Ade ${ }^{3}$, A. N. Bender ${ }^{4}$, B. A. Benson ${ }^{5}$, F. Bertoldi ${ }^{1}$, H.-M. Cho ${ }^{6}$, G. Chon ${ }^{1,2}$, \\ J. Clarke ${ }^{5}$, M. Dobbs ${ }^{7}$, D. Ferrusca ${ }^{5}$, N. W. Halverson ${ }^{4}$, W. L. Holzapfel ${ }^{5}$, C. Horellou ${ }^{8}$, D. Johansson ${ }^{8}$, J. Kennedy $^{7}$, \\ Z. Kermish ${ }^{5}$, R. Kneissl ${ }^{2}$, T. Lanting ${ }^{7,9}$, A. T. Lee ${ }^{5,10}$, M. Lueker ${ }^{5}$, J. Mehl ${ }^{5}$, K. M. Menten ${ }^{2}$, T. Plagge ${ }^{5}$, \\ C. L. Reichardt ${ }^{5}$, P. L. Richards ${ }^{5}$, R. Schaaf ${ }^{1}$, D. Schwan ${ }^{5}$, H. Spieler ${ }^{10}$, C. Tucker ${ }^{3}$, A. Weiss ${ }^{2}$, and O. Zahn ${ }^{5}$ \\ 1 Argelander Institute for Astronomy, Bonn University, Bonn, Germany \\ e-mail: mnord@astro.uni-bonn.de \\ 2 Max Planck Institute for Radioastronomy, 53121 Bonn, Germany \\ 3 School of Physics and Astronomy, Cardiff University, CF24 3YB Wales, UK \\ 4 Center for Astrophysics and Space Astronomy, University of Colorado, Boulder, CO, 80309, USA \\ 5 Department of Physics, University of California, Berkeley, CA, 94720, USA \\ ${ }^{6}$ National Institute of Standards and Technology, Boulder, CO, 80305, USA \\ 7 Physics Department, McGill University, H2T 2Y8 Montreal, Canada \\ 8 Onsala Space Observatory, Chalmers University of Technology, 43992 Onsala, Sweden \\ 9 D-Wave Systems Inc., Burnaby, V5C 6G9, Canada \\ ${ }^{10}$ Lawrence Berkeley National Laboratory, Berkeley, CA, 94720, USA
}

Received 29 January 2009 / Accepted 23 July 2009

\section{ABSTRACT}

\begin{abstract}
Context. Observations of the Sunyaev-Zel'dovich effect (SZE) from galaxy clusters are emerging as a powerful tool in cosmology. Besides large cluster surveys, resolved SZE images of individual clusters can shed light on the physics of the intra-cluster medium (ICM) and allow accurate measurements of the cluster gas and total masses.

Aims. We used the APEX-SZ and LABOCA bolometer cameras on the APEX telescope to map both the decrement of the SZE at $150 \mathrm{GHz}$ and the increment at $345 \mathrm{GHz}$ toward the rich and X-ray luminous galaxy cluster Abell 2163 at redshift 0.203 . The SZE images were used, in conjunction with archival XMM-Newton X-ray data, to model the radial density and temperature distribution of the ICM, as well as to derive the gas mass fraction in the cluster under the assumption of hydrostatic equilibrium.

Methods. We describe the data analysis techniques developed to extract the faint and extended SZE signal. We used the isothermal $\beta$ model to fit the SZE decrement/increment radial profiles. We performed a simple, non-parametric de-projection of the radial density and temperature profiles, in conjunction with X-ray data, under the simplifying assumption of spherical symmetry. We combined the peak SZE signals derived in this paper with published SZE measurements of this cluster to derive the cluster line-of-sight bulk velocity and the central Comptonization, using priors on the ICM temperature.

Results. We find that the best-fit isothermal model to the SZE data is consistent with the ICM properties implied by the X-ray data, particularly inside the central $1 \mathrm{Mpc}$ radius. Inside a radius of $\sim 1500 \mathrm{kpc}$ from the cluster center, the mean gas temperature derived from our SZE/X-ray joint analysis is $10.4 \pm 1.4 \mathrm{keV}$. The error budget for the derived temperature profile is dominated by statistical errors in the $150 \mathrm{GHz}$ SZE image. From the isothermal analysis combined with previously published data, we find a line-of-sight peculiar velocity consistent with zero; $v_{r}=-140 \pm 460 \mathrm{~km} \mathrm{~s}^{-1}$, and a central Comptonization $y_{0}=3.42 \pm 0.32 \times 10^{-4}$ for Abell 2163 . Conclusions. Although the assumptions of hydrostatic equilibrium and spherical symmetry may not be optimal for this complex system, the results obtained under these assumptions are consistent with X-ray and weak-lensing measurements. This shows the applicability of the simple joint SZE and X-ray de-projection technique described in this paper for clusters with a wide range of dynamical states.
\end{abstract}

Key words. galaxies: clusters: individual: Abell 2163 - cosmic microwave background - cosmology: observations

\section{Introduction}

The Sunyaev-Zel'dovich effect (SZE, Sunyaev \& Zel'dovich 1970; Birkinshaw 1999) provides a powerful probe of the largescale structure in the Universe by imprinting the thermal energy of clusters of galaxies on the cosmic microwave background (CMB). The inverse Compton scattering of CMB photons by the hot intra-cluster medium (ICM) has a characteristic frequency dependence and a power spectrum that is very different from that of the primary CMB anisotropies.

A number of blind SZE imaging surveys are in progress (e.g. Ruhl et al. 2004; Kosowsky 2006) with the aim of tracing the large-scale structure of the Universe through the detection of galaxy clusters, and the first blind SZE detections have recently been reported (Staniszewski et al. 2009).

With sufficiently high resolution, the SZE can be used to map the pressure structure of individual clusters. Once combined with information on the X-ray surface brightness, the SZE thus provides constraints on gas temperatures and total mass distributions inside clusters. Such constraints are free of the potential biases of X-ray spectroscopy. A further comparison with weaklensing maps yields insight into the dynamical state of the cluster by checking the validity of the hydrostatic equilibrium condition. Joint de-projection methods using X-ray and SZE maps have 
been proposed (e.g. Lee \& Suto 2004; Puchwein \& Bartelmann 2006), but so far their implementation using real SZE data has been limited to unresolved or barely resolved SZE maps (e.g. De Filippis et al. 2005). Kitayama et al. (2004) presented the first tempertaure deprojection of a cluster using a combined SZE and X-ray analysis. However, this analysis was limited to using a parametric model for the SZE signal. One main objective of this paper is to show the potential of such a de-projection analysis using high signal-to-noise resolved SZE maps without the limitations of parametric models.

Abell 2163 is a hot, X-ray luminous galaxy cluster at $z=$ 0.203 , with a mean X-ray temperature in the central region of $T_{\mathrm{X}}=12_{-1.1}^{+1.3} \mathrm{keV}$ (Markevitch \& Vikhlinin 2001). It has a relatively large angular extent, with a virial radius estimated from weak-lensing mass modeling to be on the order of $15 \mathrm{arcmin}$ (Radovich et al. 2008), making the primary CMB anisotropies a major source of systematic uncertainty in the SZE measurements. Detailed X-ray observations suggest that Abell 2163 is a merger system (Elbaz et al. 1995; Govoni et al. 2004), with asymmetric X-ray temperature structures and strong radio halos. The merger scenario is also supported by optical observations, most notably from the presence of two bright $\mathrm{cD}$ galaxies (BCGs; Maurogordato et al. 2008). The cluster has been observed in the SZE at $30 \mathrm{GHz}$ with OVRO/BIMA (Reese et al. 2002), and at 142, 217, and $268 \mathrm{GHz}$ using SuZIE (Holzapfel et al. 1997a,b). Using relativistic corrections to the SZE, Hansen et al. (2002) used these data to directly constrain the mean ICM gas temperature in Abell 2163, albeit with very large uncertainties.

During the commissioning observation of the APEX-SZ bolometer camera (Schwan et al. 2003; Dobbs et al. 2006) in 2007, we detected Abell 2163 with high significance $(12 \sigma)$ at $150 \mathrm{GHz}$ with arcminute resolution. We observed the cluster in the SZE increment at $345 \mathrm{GHz}$ using the LABOCA bolometer camera (Kreysa et al. 2003; Siringo et al. 2009) on APEX, providing images with an angular resolution of 19.5".

With a map larger than $12^{\prime}$ across, the present LABOCA data on Abell 2163 provide the first large-area imaging of a galaxy cluster at sub-mm wavelengths. High-resolution SZE increment imaging is important in view of planned future observations of clusters at sub-mm wavelengths, and for controlling potential foregrounds. However, due to limitations in constraining the SZ emission at large radii, the use of the sub-mm data in this paper is limited to the application of the multi-frequency SZE measurement to model the SZE spectrum and derive constraints on the thermal and kinematic SZE components.

In Sects. 2 and 3 we describe the observations and data reduction algorithms used to obtain maps at 150 and $345 \mathrm{GHz}$. We also describe the analysis of complementary XMM-Newton data. In Sect. 4, a simple isothermal model of the cluster gas density distribution is presented. A joint SZE/X-ray de-projection of the cluster temperature and density structure is performed in Sect. 5. Finally, we incorporate all available SZE observations of Abell 2163 in Sect. 6 to derive the best-fit line-of-sight peculiar velocity and central Comptonization from the SZE spectrum. We list our conclusions in Sect. 7.

\section{Observations}

\subsection{APEX-SZ observations at $150 \mathrm{GHz}$}

Observations of Abell 2163 at $150 \mathrm{GHz}$ were carried out in April 2007 with the APEX-SZ bolometer camera, with a measured beam full-width half-maximum (FWHM) of $58^{\prime \prime}$ (determined from observations of Mars) and an effective bandwidth of $23 \mathrm{GHz}$ (Halverson et al. 2008, hereafter H09). At the time of observation, 280 of the 330 bolometers were read out, and of these about 160 were used for the map making after applying a noise cutoff (Sect. 3).

To obtain a uniform coverage over the extended emission of the cluster, horizontal raster scans were made with a $15^{\prime} \times$ $15^{\prime}$ scan pattern, tracking the central cluster position. The median scan speed was $230^{\prime \prime} \mathrm{s}^{-1}$, and the total integration time was $7.6 \mathrm{~h}$. The observing conditions were good to moderate for the site, with $150 \mathrm{GHz}$ zenith opacities in the range 0.04-0.09.

To test a different scan strategy, $0.8 \mathrm{~h}$ of additional observations were carried out in August 2007, scanning in circles of radius 6 arcmin. At the beginning of each 2-min subscan, the circle center was placed on the cluster center, and during the subscan the source was allowed to drift through the scan pattern.

\section{2. $L A B O C A$ observations at $345 \mathrm{GHz}$}

After producing a high signal-to-noise detection with APEX-SZ, five hours of follow-up observations at $345 \mathrm{GHz}$ with LABOCA were completed in September 2007. Seven further hours of observations at this frequency were carried out in May 2008.

At the time of observation, LABOCA had 268 optically active bolometer channels, of which around 240 were used for mapping of Abell 2163. From observations of Neptune the coadded beam size was determined to be 19.5" (FWHM). The observing conditions were stable in both observing periods, with a typical atmospheric zenith opacity of $0.2-0.3$ at the observing frequency. Abell 2163 was observed in an elevation range of 40-70 degrees.

For the LABOCA observations, a spiral pattern was used with inner and outer radii of 120 and 180 arcsec, respectively. Each scan was made up of four such spirals, separated by 140 arcsec in azimuth and elevation, to make the coverage close to constant over the central part of the cluster.

\subsection{X-ray observations}

$\mathrm{X}$-ray emission originating from clusters provides additional information on their ICM, and in the interpretation of our results we make use of archival XMM observations of Abell 2163. The cluster was observed in August 2000 as part of the guaranteed time from the first year of operation. Due to the large size of the target, as compared to the XMM field of view, a mosaic of five pointings was required to fully cover the cluster: one "onsource" pointing ${ }^{1}$ and four offset pointings ${ }^{2}$ to probe the outskirts of the cluster and the surrounding cosmic background. The nominal exposure time of each observation was about $30 \mathrm{ks}$. Since the full exposure could not be achieved for the "on-source" pointing, the observation was completed with a second pointing ${ }^{3}$ in September 2001, which is included in our analysis.

\section{Data reduction, calibration and mapping}

\subsection{General sky-noise considerations}

The SZE signal from Abell 2163 is extended over more than 20 arcmin, which is comparable to the field-of-view of APEX-SZ (23' across) as well as that of LABOCA (11' across).

\footnotetext{
1 Observation Id: 0112230601.

2 ObsId: 0112230701, 0112230801, 0112230901, 0112231001.

3 ObsId: 0112231501.
} 
This makes the subtraction of atmospheric signals very difficult; subtracting common-mode signals across the array and applying polynomial baselines (corresponding to large-scale Fourier modes) in this case has the side effect of also removing astronomical signals on scales comparable to the field-of-view of the array.

The properties of the two instruments used in this analysis differ substantially, calling for highly specialized reduction schemes in each case. In general, the APEX-SZ observations were carried out in poorer observing conditions than the LABOCA observations, with relatively high levels of precipitable water vapor. For this reason, the APEX-SZ data suffers from excess low-frequency noise correlated on scales smaller than the array, requiring high-pass filtering of individual bolometer time streams to be applied after removing the correlated atmospheric signal. While this step enhances the signal-to-noise ratio of the detection, it also removes additional astrophysical signal. To account for this, we make use of a point source transfer function as in H09. It is used as follows: (i) for the isothermal analysis, we model the cluster by convolving the parameterized cluster model with the transfer function and compare the result with our reduced map; and (ii) for the non-parametric non-isothermal analysis, we deconvolve the reduced map with the transfer function.

In spite of the higher frequency band, there is no excessive low-frequency atmospheric noise component in the time streams of the LABOCA data. However, because of the lower sensitivity of this instrument with respect to the SZE signal, a smaller scan pattern was used, compared to the APEX-SZ observations, to maximize the signal-to-noise ratio in the limited time available. The small scan pattern fundamentally limits the scale on which the SZE signal can be recovered. In addition, the details of the LABOCA data reduction limits the use of a transfer function since the latter cannot be constructed to be linear; in other words, the reduction pipeline acts differently on a point source and an extended source. To recover as much of the SZE increment signal as possible, we use a slightly modified form of the iterative map-making algorithm outlined by Enoch et al. (2006).

For the complete reduction, the bolometer array data analysis software $\mathrm{BoA}^{4}$ has been used.

\subsection{Calibration of the millimeter data}

Primary flux calibration for APEX-SZ and LABOCA relies on observations of planets. The APEX-SZ data is calibrated with daily raster scans of Mars on all the 280 elements of the array (cf. H09). From these observations the gain, beam shape and position on the sky are determined for each bolometer. After adjusting the bolometer relative calibrations and angular offsets derived from the Mars observations, a co-added rms weighted map is made using all optically active bolometers. This map is used to determine the overall calibration factor, taking into account the significant side lobes of the beam.

The calibration procedure is similar for LABOCA, but here the primary calibrator is Neptune, which remains unresolved in the 19.5" beam. Because Neptune was not always observable, several secondary calibrators were used for LABOCA (Siringo et al. 2009). These sources used have been monitored by the LABOCA team since early 2007, and their $345 \mathrm{GHz}$ flux densities, relative to Neptune, are known to within about $6 \%$.

The absolute flux of Mars at the APEX-SZ frequency at each observing period is determined using a modification of the Rudy

${ }^{4}$ http://www . apex-telescope.org/bolometer/laboca/boa/ model (Rudy et al. 1987; Muhleman \& Berge 1991), maintained by Bryan Butler ${ }^{5}$ and corrected to be in agreement with recent WMAP results as described in detail in H09. The absolute flux of Neptune at $345 \mathrm{GHz}$ is derived from cross-calibrations of Neptune against Mars (Griffin \& Orton 1993).

To monitor the stability of opacity corrections at different elevations, secondary-calibrator measurements are used. The computation of opacities relies on skydip measurements combined with tau-meter readings (Weiss et al. 2008; Siringo et al. 2009).

\subsection{APEX-SZ data reduction}

Time stream data from APEX-SZ is processed through a data reduction pipeline and binned to form maps. This process is described here. The pipeline is slightly different for raster scans and circular drift scans, but identical for each scan within these two subsets.

After eliminating detectors with low optical response, correlated (atmospheric and electronic) noise is removed by subtracting the median signal from across the good channels of the array at each time sample. Individual time streams are then despiked by flagging and removing data that deviates from the baseline by more than $5 \sigma$, which typically corresponds to less than $0.1 \%$ of the data. Electronic glitches are recognizable because they occur with durations shorter than the detector response time. These features, seen only in a negligible amount of data, are removed as well.

To baseline the data of a raster scan, the scan is divided into subscans of constant elevation, resulting in subscans extending over $30^{\prime}$ in azimuth. Turnarounds in the scan pattern, where the scan speed is significantly lower, are flagged and removed. A fifth order polynomial is fitted to and subtracted from each constant-elevation subscan, effectively high-pass filtering the time streams.

Circular drift scans are baselined by defining subscans consisting of 2.5 full circles and applying a fifth order polynomial, after flagging the first full circle of the scan (which usually contains intervals of high scan acceleration). This polynomial baseline corresponds to a spatial filter similar to that applied to the raster scans. Simultaneously, an airmass correction is applied as described in $\mathrm{H} 09$.

For each scan, a map with $10^{\prime \prime} \times 10^{\prime \prime}$ sized pixels is constructed, weighting the data by the inverse rms of each reduced time stream. All maps are then co-added. In parallel with the reduction of the data, a beam-shaped source, without noise and translated into time stream data, is passed through an identical pipeline to obtain the point source transfer function, as described by H09. All flags and weights on the data are carried over to the artificial data.

The resulting transfer function is used to deconvolve the high significance co-added map of Abell 2163 to the intrinsic resolution of the instrument. The deconvolution is done iteratively, and is similar to the CLEAN algorithm for interferometry data (Högbom 1974; Schwarz 1978) in that the source is modeled as a sum of many point sources; here, however, the process is carried out in map space rather than Fourier space. The raw (pipeline filtered) map, $M_{0}$, is first convolved with the beam to reduce the sample variance on small spatial scales. Each pixel in the map is then divided by the local rms to create a signal-to-noise map, $N_{0}$, from which the brightest pixel is selected. The corresponding point source flux corresponding to this peak pixel value is computed, taking into account both the beam smoothing of $M_{0}$ and

\footnotetext{
5 http://www.aoc.nrao.edu/bbutler/work/mars/model
} 


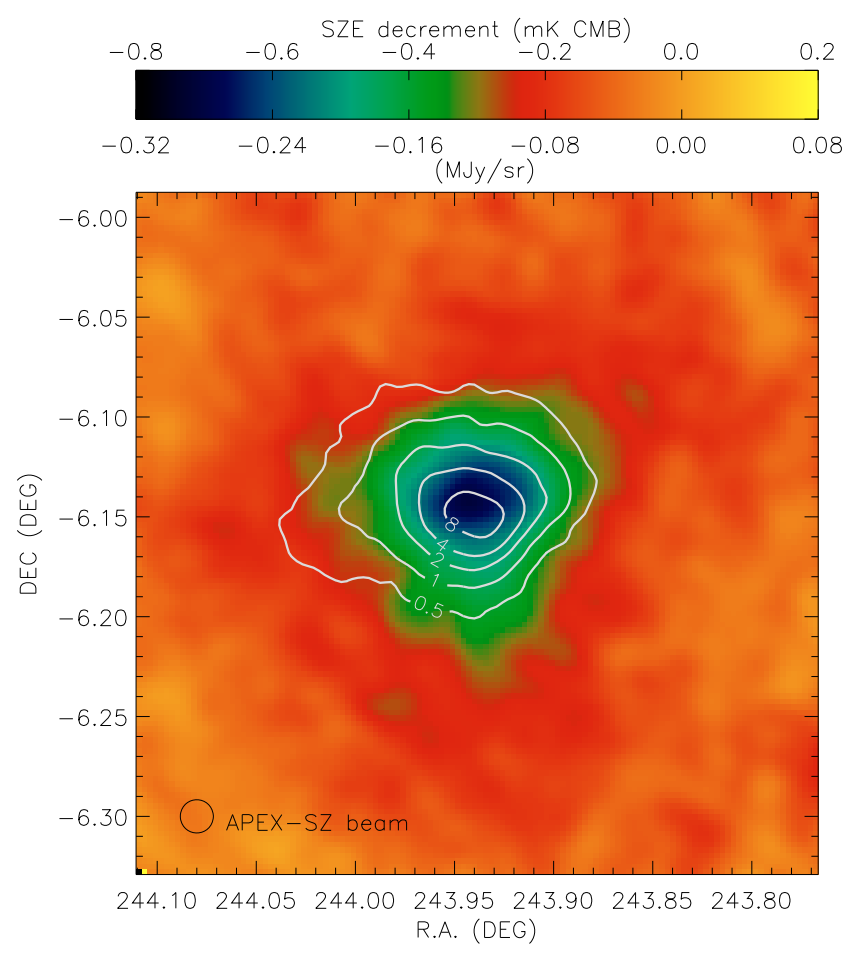

Fig. 1. Map of Abell 2163 at $150 \mathrm{GHz}$, overlaid with XMM-Newton X-ray contours (see Fig. 3) in units of $10^{-13} \mathrm{erg} \mathrm{s}^{-1} \mathrm{~cm}^{-2} \operatorname{arcmin}^{-2}$. Because the correlated-noise removal has attenuated the source signal, the map has been de-convolved using the point source transfer function (see text).

the complete description of the inherent resolution and the effects of the reduction described by the point source transfer function. The resulting point source is re-convolved with the transfer function to represent how the point source is reflected in the raw map. This component is subtracted from $M_{0}$ to yield $M_{1}$, from which a new smoothed signal-to-noise map $N_{1}$ is constructed. The point source is also convolved with the "clean" beam and added to a map $C$ of "clean component". Next, the brightest pixel in $N_{1}$ is located, and the process is repeated until $M_{i}$ is consistent with noise. The criterion for the latter is that as many negative as positive pixels are selected in any consecutive 20 iterations. At this point, $M_{i}$ is added to the cumulative map $C$ to account for emission which has not been successfully removed as point sources. Details on the limitations of this deconvolution technique will be given in a future publication (Nord et al., in preparation). The de-convolved APEX-SZ map of Abell 2163 is shown in Fig. 1.

To compute the noise on the beam scale, we smooth the final map with the APEX-SZ beam (FWHM 58" and computing the rms in square regions with side five times the beam. In this way, we find a noise rms value of $32 \mu \mathrm{K}_{\mathrm{CMB}}$ on the beam scale, corresponding to $0.013 \mathrm{MJy} \mathrm{sr}^{-1}$, in the central region of the deconvolved map.

\section{4. $L A B O C A$ data reduction}

Because the LABOCA time streams are more stable at low frequencies than those from APEX-SZ, the data do not have to be further filtered after standard correlated-noise removal. Aside from this difference, the time stream reduction sequence used in each iteration is quite similar to that used for the APEX-SZ data, and basically follows the method outlined by Weiss et al. (2008) and detailed by Siringo et al. (2009). Maps are constructed with $6^{\prime \prime} \times 6^{\prime \prime}$ pixels, and co-added in the same way as for the APEX-SZ data.

To account for sky signal attenuation in the time stream reduction, we follow the iterative approach of Enoch et al. (2006), with one important modification. Using a suitably chosen time stream reduction algorithm, a map is produced. Each pixel with a significance of less than 3 sigma is set to zero, and the rest of the map is chosen as the template to be subtracted directly from the time streams before running the same time stream reduction yet again, and so on. While Enoch et al. take a conservative approach and derive the next template from the residual signal, we add the template back into the data prior to mapping and derive this template from the total map. This ensures that any significant feature in the current best guess of the source flux distribution is carried over to the next iteration step.

The iterative mapping algorithm requires 8 iterations before convergence is reached. In each iteration, an identical time series reduction is performed.

Because the iterative mapping technique is non-linear, it is not straightforward to characterize it by a filter function. In place of a transfer function, the instrument beam is used when fitting a model to the $345 \mathrm{GHz}$ data. Significant loss of signal is expected using the iterative approach. To quantify the level of bias, we use the best-fit $\beta$ model from the APEX-SZ measurement (Sect. 4) and pass is through the complete LABOCA data reduction pipeline. Although more than $30 \%$ of the signal is lost beyond $r_{500}$ (the radius at which the enclosed average matter overdensity is 500 times the mean cosmic density), we find that the signal loss within $r_{2500}$ (defined analogously, approximately $3^{\prime}$ ) is negligible compared to statistical errors. The central rms in the LABOCA map, computed on a scale of five times the LABOCA $F W H M\left(19.5^{\prime \prime}\right)$, is $1.9 \mathrm{mJy} /$ beam, corresponding to $0.24 \mathrm{MJy} \mathrm{sr}^{-1}$.

We find one significant point source in the final map (Fig. 2), likely thermal emission from a cluster galaxy or a background source lensed by the cluster. After highpass-filtering the map to remove structures larger than two beams (such as the cluster emission), we find a peak flux density of $11.8 \pm 1.9 \mathrm{mJy} / \mathrm{beam}$ for this source.

\subsection{XMM-Newton data reduction}

Here we describe the processing steps applied to the X-ray data.

We generate calibrated event-lists from the raw data of the 6 archival pointings using the standard procedures of the XMM Science Analysis System ${ }^{6}$ (SAS) and screen these lists for periods of high radiation due to soft solar proton flares. To this end, we apply the method of Pratt \& Arnaud (2002), which consists of fitting histograms of the high energy ${ }^{7}$ light curves with a Poisson law and rejecting time intervals exceeding the mean radiation level by more than $3 \sigma$. On average, this shortens the available exposure time by $15 \%$. Although the high energy signal is very sensitive to particle flares, one needs to adopt a coarse time sampling (104 s here) to ensure that the average number of counts per bin is significant. This implies that the wings of light curve jumps in increased radiation periods cannot easily be detected. To improve on our first filtering, we thus repeat the same

\footnotetext{
6 http://xmm.esac.esa.int/sas/, all the data processing for this paper relies on $\mathrm{v} 7.1 .2$.

7 More precisely [10-12] keV for the MOS detectors and [12-14] keV for the PN.
} 

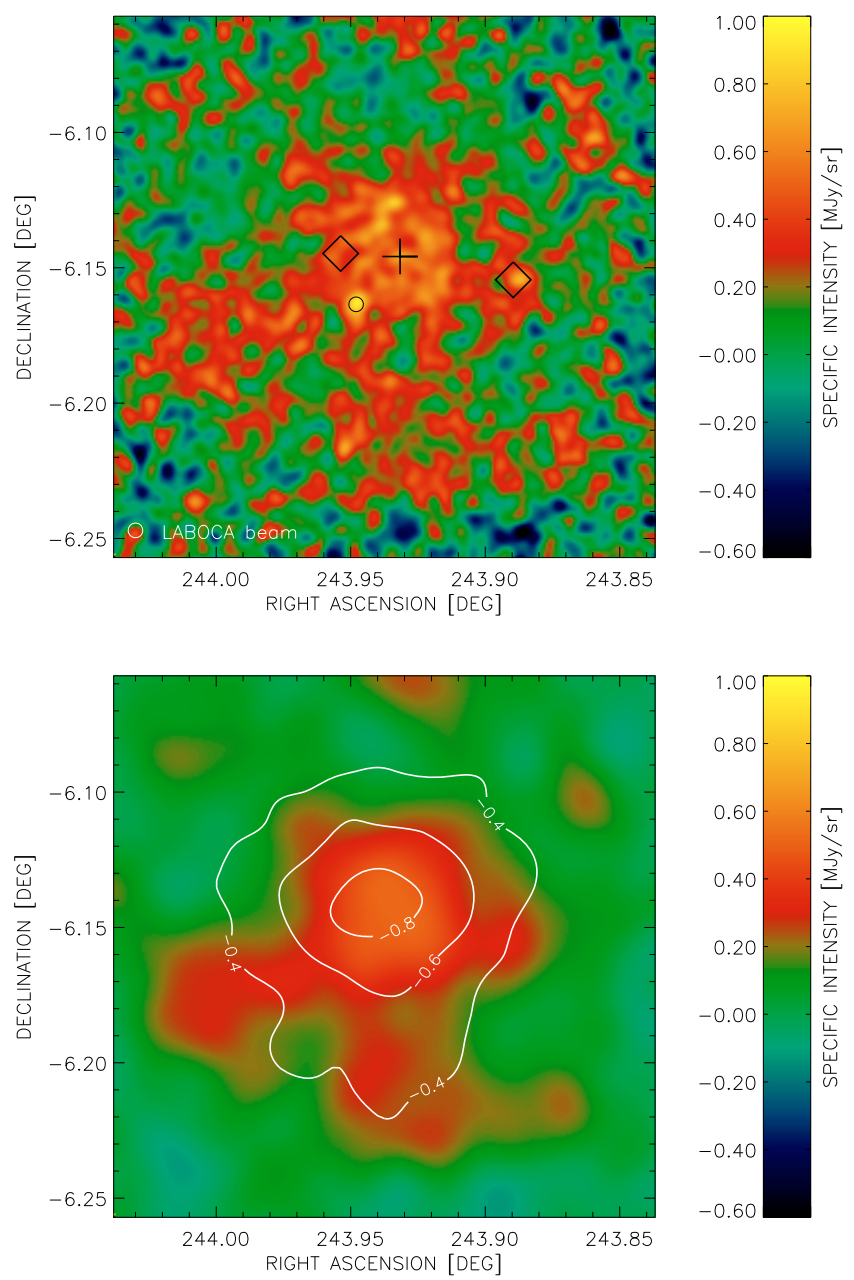

Fig. 2. Top: final $345 \mathrm{GHz}$ LABOCA map of Abell 2163, smoothed with the $19.5^{\prime \prime}$ beam. The cross marks the position of a bright flat-spectrum radio source (Cooray et al. 1998). The diamonds mark the positions of two BCGs (Maurogordato et al. 2008). The circle marks the position of the bright point source found in the present data. Bottom: LABOCA map smoothed to the APEX-SZ resolution of 1 arcmin. The bright point source described in the text has been removed. The APEX-SZ $150 \mathrm{GHz}$ map is shown as contours.

analysis on a broad low energy band, namely [0.3-10] keV with narrow time bins of $13 \mathrm{~s}$, resulting in the exclusion of an extra $3 \%$ of the data. Out-of-time event lists are also generated using the simulation mode of the SAS tasks emchain/epchain and filtered using the same time intervals.

From the filtered event lists, images and exposure maps are generated in the [0.5-2] keV band and gathered into two large mosaics. The signal to noise of the cluster in this band is about $80 \%$ of the best achievable value using the XMM bands. It is however free from the strongly varying instrumental lines around 6-7 keV and calibration uncertainties at lower energies. Combined with the weak dependence of the cooling function on temperature at low energies (soft X-rays), this makes it a very suitable band for our purpose. Since the XMM response is almost flat in this narrow band, the exposure maps are evaluated at a single average energy of $1.25 \mathrm{keV}$.

The main issue in the X-ray analysis of extended source is an accurate background modeling. To this end, we make use of the Filter Wheel Closed (FWC) dataset provided by the XMM

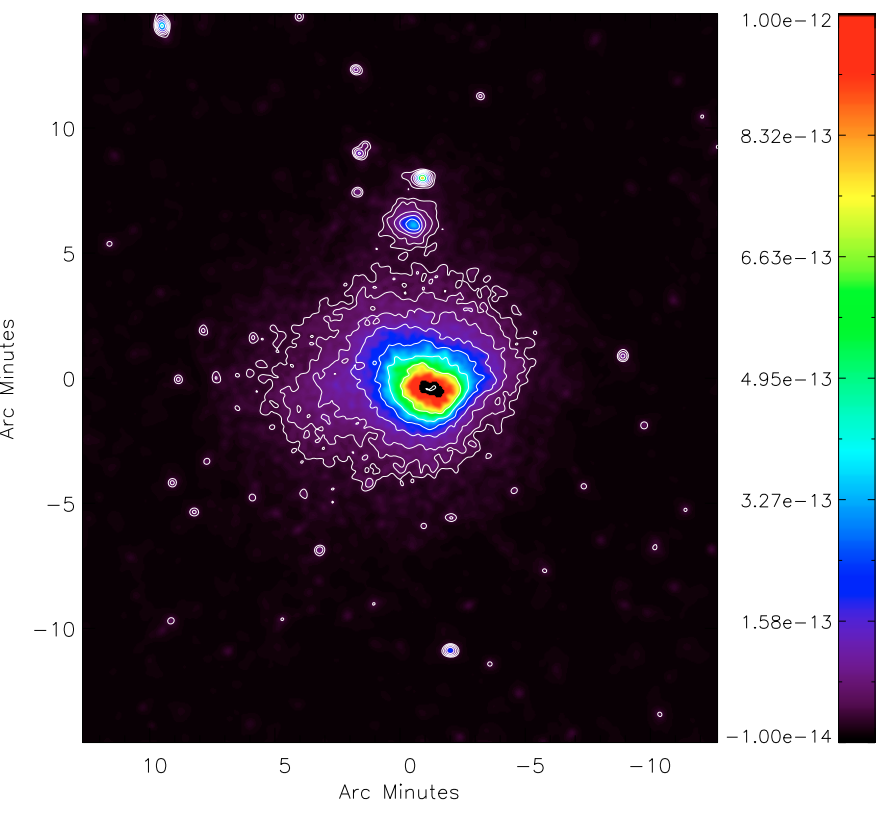

Fig. 3. Smoothed, background subtracted, X-ray map of Abell 2163 in the [0.5-2] keV band (see text for details). Logarithmically spaced contours highlight the broad dynamical range of the cluster emission. The unit of the color scale is erg s $\mathrm{s}^{-1} \mathrm{~cm}^{-2} \operatorname{arcmin}^{-2}$.

background working group ${ }^{8}$. Our model consists of a sum of a re-scaled FWC image (which represents the instrumental noise), an homogeneous cosmic background of constant surface brightness (since the area of the mosaic remains small) and a properly scaled image of the out-of-field events. The scaling of the instrumental background is allowed to vary with each pointing and instrument while the cosmic background level is just a function of the detector (to account for the different responses). Using the Cash statistics (Cash 1979), the model parameters are fitted simultaneously to the 6 observations including the data from the out of field corners and excluding the positions closer than $17^{\prime}$ from the cluster center.

In order to detect and mask the surrounding AGNs, we adaptively smooth the background subtracted raw mosaic, using the criterion of having more than 10 counts per cell. The resulting map is then exposure corrected and smoothed again by a $\sigma=2^{\prime \prime}$ Gaussian ${ }^{9}$. A source catalog is extracted from this image using SExtractor (Bertin \& Arnouts 1996) and the associated source-segmentation map is combined with an exposure time threshold in order to derive a global mask.

The tasks of background estimation and source detection are somewhat intricate, especially in the case of mosaics where steep instrumental and particle background variations can occur in between pointings. To tackle this issue, we iteratively re-perform the background modeling and AGN extraction using the updated AGN mask for the background estimate. Both products stabilize after three iterations.

The final background subtracted, adaptively smoothed image of the cluster is shown in Fig. 3.

\footnotetext{
${ }^{8}$ http://xmm2 .esac.esa.int/external/xmm_sw_cal/ background/

9 This hybrid smoothing allows to wash out the Poisson fluctuations in regions devoid of source signal without degrading the resolution over moderately bright sources (which are both the advantage and drawback of adaptive filtering alone).
} 
Table 1. $\beta$ model fit results at 150 and $345 \mathrm{GHz}$.

\begin{tabular}{lcccc}
\hline \hline Parameter & $150 \mathrm{GHz}($ elliptical $)$ & $150 \mathrm{GHz}($ spherical $)$ & $345 \mathrm{GHz}($ spherical $)$ & X-ray(elliptical) \\
\hline$X_{0}($ Central RA [J2000]) & $16^{\mathrm{h}} 15^{\mathrm{m}} 45.1^{\mathrm{s}} \pm 9^{\prime \prime}$ & $16^{\mathrm{h}} 15^{\mathrm{m}} 45.6^{\mathrm{s}} \pm 8^{\prime \prime}$ & $\left(16^{\mathrm{h}} 15^{\mathrm{m}} 45.6^{\mathrm{s}}\right)^{b}$ & $16^{\mathrm{h}} 15^{\mathrm{m}} 46.6^{\mathrm{s}} \pm 1.5^{\prime \prime}$ \\
$Y_{0}($ Central Dec [J2000]) & $-06^{\circ} 08^{\prime} 31^{\prime \prime} \pm 8^{\prime \prime}$ & $-06^{\circ} 08^{\prime} 28^{\prime \prime} \pm 8^{\prime \prime}$ & $\left(-06^{\circ} 08^{\prime} 28^{\prime \prime}\right)^{b}$ & $-06^{\circ} 08^{\prime} 44.1^{\prime \prime} \pm 1.4^{\prime \prime}$ \\
Peak signal $^{a}$ & $-0.301 \pm 0.033$ & $-0.302 \pm 0.033$ & $0.613 \pm 0.089$ & $1.075 \times 10^{-12} \pm 7 \times 10^{-15}$ \\
$\theta_{\mathrm{c}}$ (core radius) & $123 \pm 19^{\prime \prime}$ & $129 \pm 21^{\prime \prime}$ & $89 \pm 29^{\prime \prime}$ & $90.7 \pm 2.8^{\prime \prime}$ \\
$\beta$ (power-law index) & $0.85 \pm 0.14$ & $0.85 \pm 0.14$ & $(0.85)^{b}$ & $0.639 \pm 0.03$ \\
$\Phi$ (inclination angle) & $128 \pm 24^{\circ}$ & $(0.0)^{b}$ & $(0.0)^{b}$ & $1.2 \pm 6^{\circ}$ \\
$\eta$ (axial ratio) & $0.77 \pm 0.11$ & $(1.0)^{b}$ & $(1.0)^{b}$ & $0.850 \pm 0.05$ \\
\hline
\end{tabular}

${ }^{a}$ Brightness in units of MJy sr${ }^{-1}$ (SZE), and in units of $\mathrm{erg} \mathrm{s}^{-1} \mathrm{~cm}^{-2} \operatorname{arcmin}^{-2}$ (X-ray); ${ }^{b}$ fixed parameters indicated by parentheses.

\section{Isothermal modeling of the intra-cluster gas}

In order to assess our SZE measurements quantitatively and compare them with the X-ray data, we fit the SZE measurements using the well-known isothermal $\beta$ model (Cavaliere \& Fusco-Femiano 1978). This enables us to compare the X-ray surface brightness profiles with the SZE temperature decrement/increment maps and check the agreement of our SZE measurements with the prediction from the X-ray mean values.

\subsection{Isothermal gas in hydrostatic equilibrium}

The isothermal $\beta$ model is widely used to model the gas profile in clusters under the assumption of hydrostatic equilibrium. The radial distribution of gas density is given by

$n_{\mathrm{e}}(r)=n_{\mathrm{e}}(0)\left(1+\frac{r^{2}}{r_{\mathrm{c}}^{2}}\right)^{-\frac{3}{2} \beta}$,

where $n_{\mathrm{e}}$ is the density of electrons, $r$ is the radius from the center, $r_{\mathrm{c}}$ is the core radius of the gas, and $\beta$ is the power law index.

With this model, the temperature decrement (or increment) due to the SZE takes the form

$\Delta T(\theta)=f\left(x, T_{\mathrm{e}}\right) T_{\mathrm{CMB}} y=\Delta T(\theta=0)\left(1+\frac{\theta^{2}}{\theta_{\mathrm{c}}^{2}}\right)^{(1-3 \beta) / 2}$,

where $\theta$ is the angular radius on the sky, $\Delta T$ is the thermodynamic SZE temperature decrement (or increment) and $y$ is the Comptonization parameter, given by the line-of-sight integral

$y=\int\left(\frac{k_{\mathrm{B}} T_{\mathrm{e}}}{m_{\mathrm{e}} c^{2}}\right) n_{\mathrm{e}} \sigma_{\mathrm{T}} \mathrm{d} l$,

where $\sigma_{\mathrm{T}}$ is the cross section for Thomson scattering. The frequency dependence $f\left(x, T_{\mathrm{e}}\right)$ of the SZE signal is given by

$f\left(x, T_{\mathrm{e}}\right)=\left[x \frac{e^{x}+1}{e^{x}-1}-4\right]\left(1+\delta_{x, T_{\mathrm{e}}}\right)$,

with relativistic corrections contained in $\delta_{x, T_{\mathrm{e}}}$ as described by Sazonov \& Sunyaev (1998). $x$ is the dimensionless frequency $x=\frac{h v}{k_{\mathrm{B}} T_{0}}$, reads

The analogous expression for the X-ray surface brightness

$\Delta S_{\mathrm{X}}(\theta)=S_{0}\left(1+\frac{\theta^{2}}{\theta_{\mathrm{c}}^{2}}\right)^{1 / 2-3 \beta}$,

where $S_{0}$ is the central surface brightness and the different exponent comes from the $n_{\mathrm{e}}^{2}$ dependence of X-ray emissivity.

\subsection{Elliptical $\beta$ model fit to the SZE data}

Because of the significant ellipticity in the X-ray surface brightness profile, we generalize Eq. (2) to an elliptical form given, e.g., in $\mathrm{H} 09$ as

$\Delta T_{\mathrm{SZE}}=\Delta T_{0}(1+A+B)^{(1-3 \beta) / 2}$,

where

$A=\frac{\left(\cos (\Phi)\left(X-X_{0}\right)+\sin (\Phi)\left(Y-Y_{0}\right)\right)^{2}}{\theta_{\mathrm{c}}^{2}}$,

$B=\frac{\left(-\sin (\Phi)\left(X-X_{0}\right)+\cos (\Phi)\left(Y-Y_{0}\right)\right)^{2}}{\left(\eta \theta_{\mathrm{c}}\right)^{2}}$

Here $\left(X-X_{0}\right)$ and $\left(Y-Y_{0}\right)$ are angular offsets on the sky in the right ascension (RA) and declination (Dec) directions, respectively, with respect to centroid position $\left(X_{0}, Y_{0}\right)$. The axial ratio, $\eta$, is the ratio between the minor and major axis core radii and $\Phi$ is the angle between the major axis and the RA $(X)$ direction.

An elliptical $\beta$ profile is fitted to the $150 \mathrm{GHz}$ data by convolving each model function with the point source transfer function and minimizing a $\chi^{2}$ statistic weighted by the inverse square of the local map rms. Note that the raw, not the de-convolved, map is used for this fit in conjunction with the transfer function. All map pixels within a $10^{\prime}$ radius of the X-ray center (Sect. 4.3) are considered for the fit. The results of the fit are given in Table 1.

For comparison, we also fit a spherical $\beta$ profile and find roughly consistent results. At $345 \mathrm{GHz}$, the detection of Abell 2163 is less significant, we thus fix the centroid and the value of $\beta$ to those fitted from the $150 \mathrm{GHz}$ measurement and fit for the remaining parameters.

Errors in the fitted profiles are estimated using a Monte Carlo approach. The map data is jack-knifed by inverting the sign of half the individual scan maps (randomly chosen), and the maps are co-added to generate a pure noise map. After convolving the best-fit model with the transfer function and adding the result to the noise map, the $\chi^{2}$ statistic is again minimized to find the best set of parameters for the new map. The entire procedure is repeated 1000 times, upon which the errors in the respective parameters are taken as the scatter in the distributions of their fitted values. We verify that the mean of the distributions of fitted parameters are consistent with the best-fit model.

\subsection{Elliptical $\beta$-model fit to the $X$-ray data}

To allow for a basic comparison of the SZE and X-ray data in the context of the isothermal $\beta$ model, we perform an isothermal fit to the X-ray data, similar to that used for the SZE data. As for 
the SZE analysis, we generalize the spherical $\beta$ model of Eq. (4) to the elliptical case, as:

$\Delta S_{\mathrm{X}}=\Delta S_{0}(1+A+B)^{1 / 2-3 \beta}$.

This model is fitted to the raw X-ray images using the Cash statistic (Cash 1979). The Poisson nature of the data does not allow here for a direct background subtraction; instead we add the simple two-component background described in Sect. 3.5 to the fitted components. The results of the fit are presented in Table 1.

\subsection{Systematic uncertainties}

\subsubsection{Galactic dust}

The effect of galactic dust emission on the SZE measurement of Abell 2163 has been estimated by Lamarre et al. (1998) for the PRONAOS balloon experiment and by LaRoque et al. (2002) for the SuZIE experiment (Holzapfel et al. 1997a,b). However, because these two estimates apply to scan strategies and spatial filtering functions very different from ours, it is not appropriate to extrapolate from either of them.

To estimate the dust correction at 345 and $150 \mathrm{GHz}$, we use the IRAS far-infrared $60 \mu \mathrm{m}$ and $100 \mu \mathrm{m}$ dust maps (Schlegel et al. 1998) on the Abell 2163 field. Smoothing the $60 \mu \mathrm{m}$ map to the $4.3^{\prime}$ resolution of the $100 \mu \mathrm{m}$ map and re-sampling the maps so that each pixel has a side of one arcminute, we extrapolate to the frequencies of the SZE measurements using a grey-body spectrum of dust emissivity

$$
F_{\mathrm{d}} \propto \frac{v^{3+\alpha}}{\exp \left(h v / k_{\mathrm{B}} T_{\mathrm{d}}\right)-1},
$$

where $\alpha$ is the dust spectral index. Using $\alpha=2.0$ (Finkbeiner et al. 1999) we fit to $T_{\mathrm{d}}$ for every $1^{\prime} \times 1^{\prime}$ pixel pair in the two dust maps. After smoothing the resulting temperature map with a 4.3' Gaussian kernel to remove noise artifacts, we use Eq. (7) to extrapolate the intensity at 345 and $150 \mathrm{GHz}$, normalizing at $100 \mu \mathrm{m}$.

The simplest way of removing the dust component from the data is to subtract it before any filtering is applied by the reduction. We thus create time streams corresponding to the extrapolated dust maps on the coordinates covered by APEX-SZ and LABOCA and subtract these from the raw time streams before proceeding with reductions identical to those described in Sect. 3. In this way, we find corrections of $-0.6 \%$ to the SZE decrement at $150 \mathrm{GHz}$ and $+1.8 \%$ to the SZE increment at $345 \mathrm{GHz}$. Effects on the other fitted parameters are negligible. We assume that contamination on angular scales smaller than four arcminutes (i.e. not resolved by IRAS) are also negligible.

\subsubsection{Point source contamination}

After subtracting a preliminary (possibly point source contaminated) $\beta$ model from each map, we smooth the maps to the respective beam scales and look for point source detections above $3 \sigma$. One source (cf. Fig. 2) is found in the LABOCA map at $345 \mathrm{GHz}$. No sources are found in the APEX-SZ $150 \mathrm{GHz}$ map. Point sources which are detected with sufficient $S / N$ in the maps could be removed by subtracting fitted Gaussians. Here we take a somewhat more conservative approach and simply disregard a region around the $345 \mathrm{GHz}$ point source in a subsequent fit with a new $\beta$ model.

Extragalactic infrared point sources are not expected to correlate spatially with clusters of galaxies, and a distribution of sources can be well approximated by excess noise in any differential measurement (White \& Majumdar 2004), thus having little systematic effect on bolometric SZE measurements.

Although lensing by a massive cluster such as Abell 2163 can significantly increase the number of detected sources in the cluster central region, this effect conserves the total intensity of the background and only becomes important when faint sources are raised above the detection limit and removed, and the unresolved background intensity is thereby systematically lowered (e.g. Loeb \& Refregier 1997). It is not clear whether the bright point source found in the $345 \mathrm{GHz}$ map is a lensed background source; however, we have verified that the effect in fitted parameters of excluding the region around this source for the fit is merely $4 \%$, i.e. smaller than both the derived statistical errors and the absolute calibration uncertainty.

Radio sources are expected to correlate strongly with clusters of galaxies (e.g. Reddy \& Yun 2004). We have searched the NVSS catalog (Condon et al. 1998) for sources brighter than $5 \mathrm{mJy}$ at $1.4 \mathrm{GHz}$. The flux of each source is extrapolated to $150 \mathrm{GHz}$ using the spectral index maps of Feretti et al. (2004). With this simple approach, even the brightest source will have a peak flux considerably lower than the rms in the APEX-SZ map. Cooray et al. (1998) report an inverted-spectrum non-thermal source of $3 \mathrm{mJy}$ at $30 \mathrm{GHz}$, indicated in Fig. 2. No counterpart can be seen in either SZE map. High-pass filtering the APEX-SZ map, after subtracting the best-fit model of the cluster, to remove all structure larger than $3^{\prime}$ results in an effective rms of $30 \mu \mathrm{K}_{\mathrm{CMB}}$ at the position of this source. The fact that the source is not seen in this filtered map allows us to put a lower limit on the spectral index as $\alpha_{30}^{150} \gtrsim 0.5\left(S_{v} \propto v^{-\alpha}\right)$. Subtracting the corresponding signal from the APEX-SZ map before the $\beta$ model fit results in a systematic shift in the central SZE decrement of less than $1 \%$. Based on these considerations, we consider systematic effects from radio sources negligible in comparison to primary CMB anisotropies and absolute calibration.

\subsubsection{Primary $\mathrm{CMB}$ contamination}

For a very extended cluster like Abell 2163, with an estimated $r_{500}$ greater than $7^{\prime}$ (Radovich et al. 2008), the temperature anisotropies in the CMB are a potential source of confusion in the SZE measurements. This uncertainty particularly affects the kinematic SZE signal because the latter has a frequency dependence identical to that of the CMB thermal spectrum. Note that this is not an issue for CMB power on scales $\gtrsim 15^{\prime}$ due to the indirect high-pass filtering of our maps (through polynomial baselines in the bolometer time series).

To quantify the level of contamination on scales comparable to the SZE emission, the HEALpix software (Gorski et al. 2005) is used to generate 100 realizations of the CMB sky in a one square degree field with a resolution of $1.7^{\prime}$. The input power spectrum for this generation is computed using the CMBfast code (Zaldarriaga \& Seljak 2000) with the WMAP 5-year cosmology (Komatsu et al. 2009). Relying on the results of LaRoque et al. (2002), diffuse secondary CMB anisotropies other than the SZE are considered negligible.

Each primary-CMB map is re-sampled as time streams corresponding to a typical scan of the SZE observations at the two frequencies and run through the reduction pipelines to account for filtering. The $\beta$ model fit is repeated after subtracting each of the thus filtered primary-CMB realizations. The scatter in the results of each parameter is taken as the $68 \%$ systematic marginal error from primary $\mathrm{CMB}$ contamination. We find a fractional error of $3.4 \%$ at $150 \mathrm{GHz}$ and $2.9 \%$ at $345 \mathrm{GHz}$. 
The primary-CMB component has been simulated on the relevant scales compared to the cluster emission, with scales smaller than $1.7^{\prime}$ not taken into account. This is justified because the power of the primary anisotropies drops rapidly on scales smaller than a few arcminutes.

\subsubsection{Pipeline filter function}

To test how well the point source transfer function represents the filtering of the SZE signal in map space at $150 \mathrm{GHz}$, we carry out a series of reductions of known signals. To this end, we remove astrophysical signals from the data by dividing the scans into two minute segments and randomly inverting half of these. Before passing the noise data through the pipeline, a beam-convolved $\beta$ model is added to the time streams. The point source transfer function is used to reconstruct the $\beta$ model after reduction, and the procedure is repeated for a large number of $\beta$ models in the range $50^{\prime \prime} \leq \theta_{\mathrm{c}} \leq 150^{\prime \prime}, 0.5 \leq \beta \leq 1.4$. We find no evidence that the level of noise affects the result other than to increase the scatter in fitted parameters; we thus use bright model sources $\left(\sim 10 \mathrm{mK}_{\mathrm{CMB}}\right.$ at $\left.150 \mathrm{GHz}\right)$ to estimate the effect of the pipeline.

Although the fitted central amplitude can be systematically reduced by as much as $12 \%$ for combinations of small $\beta$ and large $\theta_{\mathrm{c}}$ (characterizing very broad profiles, where some modes in the map are irrecoverable by the transfer function), we find the systematic shift to be no more than $2 \%$ for either of the models fitted to the actual $150 \mathrm{GHz}$ data. Due to the degeneracy between $\beta$ and $\theta_{\mathrm{c}}$, there is considerable scatter in these parameters when fitted to the simulated data. Keeping one parameter fixed and fitting the other, we find that the systematic errors in both parameters due to inaccuracies in modeling the pipeline filter function are negligible.

\subsection{Results of the isothermal modeling}

Figure 4 shows the radial profile of the best-fit spherical $\beta$ profile to the $150 \mathrm{GHz}$ data and the same profile convolved with the transfer function, as well as the profiles of the raw and de-convolved maps. The best-fit $\beta$ model parameters, corrected for systematic effects as described below, are given in Table 1. Although derived from a completely independent method, the radial profile of the de-convolved map is fully consistent with the beam-smoothed best-fit model within the $10^{\prime}$ truncation radius used for the fit.

Comparing the results of the X-ray and SZE fits, a nonnegligible offset $\left(21 \pm 8^{\prime \prime}\right)$, possibly caused by an asymmetric temperature distribution near the center, appears between the two centroid positions. The origin of this offset will be discussed as part of further analysis in a future publication (Kneissl et al., in preparation). The other fitted parameters show an overall consistency in the global shape of the emission. We note that in spite of the significant ellipticity in the X-ray surface brightness, the elliptical model of the SZE signal is only barely inconsistent with the spherical one (the axial ratio deviates from 1 by only $1.7 \sigma$ ). The most likely explanations for this are the poorer resolution of the SZE data which results in a greater uncertainty on the ellipticity, and the inherent fact that the SZE signal, due to the density weighting, is naturally smoother and more diffuse.

\section{Non-isothermal modeling of the intra-cluster gas}

Several authors have discussed the joint modeling of SZE and X-ray data for de-projecting ICM parameters (see, e.g.,

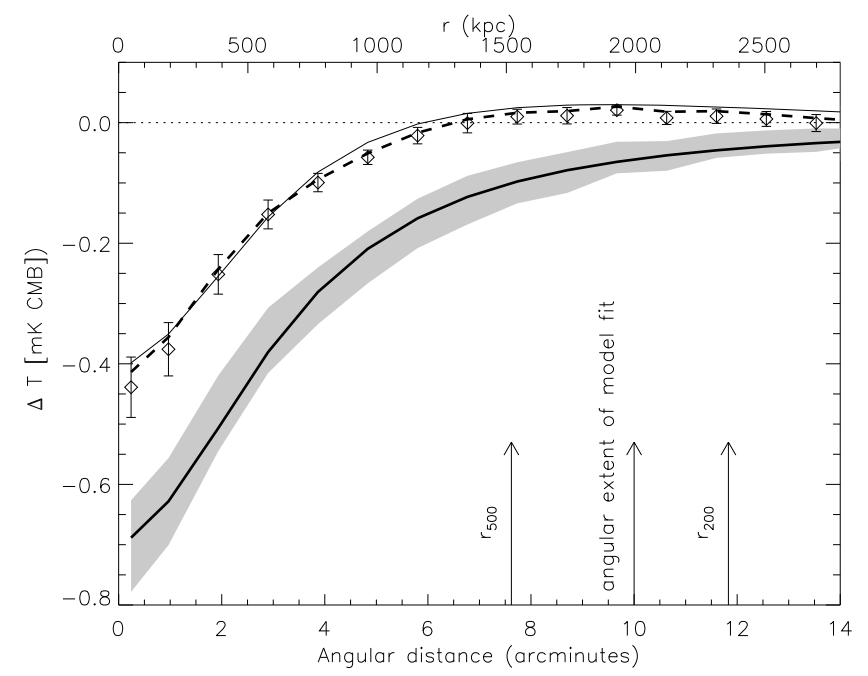

Fig. 4. Radial SZE profile of Abell 2163 at $150 \mathrm{GHz}$. Error bars indicate the profile computed from the reduction-attenuated raw map (before deconvolution; this is the map used for the parametric fit) while the shaded region represents the profile from the map de-convolved to the beam resolution (note that this map was not used to derive the best fit $\beta$ model. The profile of the best-fit isothermal $\beta$ model is indicated by solid lines; the thick line represents that model convolved with the beam, while the thin line represents the same model convolved with the transfer function. The dashed line indicates the profile of the deconvolved map, directly re-convolved with the transfer function. The vertical arrows indicates the radial cut of $10^{\prime}$ used for the model fit, as well as $r_{200}$ and $r_{500}$.

Yoshikawa \& Suto 1999; Zaroubi et al. 2001; Puchwein \& Bartelmann 2007; Ameglio et al. 2007). However, most of these analyses have been limited to analytic cluster models or numerical simulations. Our work here represents the first attempt at deprojecting cluster density and temperature profiles using actual SZE imaging data, in combination with the X-ray data, without resorting to any parametric models for the gas or dark matter distribution.

Given the large angular size of Abell 2163, the arcminute resolution of APEX-SZ is sufficient to carry out a joint radial density and temperature modeling of the ICM in combination with publicly available X-ray data. Although the LABOCA map has more than a factor of two better resolution, due to its high noise level and small field of view we restrict the analysis in this section to the APEX-SZ data.

\subsection{Method}

\subsubsection{Deprojection method}

For the deprojection analysis we use a direct inversion technique based on the Abel integral. The technique was first proposed by Silk \& White (1978; see also Yoshikawa \& Suto 1999). Apart from the assumption of spherical symmetry, there are no additional theoretical constraints (e.g. hydrostatic equilibrium or polytropic gas index), making this is a natural method for extracting ICM parameters, assuming that spherical symmetry indeed provides a good estimate of the structure. The details of this method and its limitations will be discussed in a future publication (Basu et al., in preparation); here we present the outline of the method and the results for Abell 2163. X-ray spectroscopic measurements have shown that Abell 2163 has a complicated temperature structure at the center and is most likely a merging 
system (Elbaz et al. 1995; Govoni et al. 2004). Nevertheless, the de-projection analysis with spherical symmetry implemented here shows how resolved SZE images of clusters can immediately be used in combination with X-ray data to gain a better understanding of the gas and mass distribution.

The deprojection analysis is based entirely on the radial profiles of X-ray surface brightness and SZE temperature decrement (or increment). From Eq. (2) the SZE temperature decrement can be written as the integral over the line of sight;

$\Delta T(R)=2 A_{\mathrm{SZ}} \int_{R}^{\infty} f\left(x, T_{\mathrm{e}}\right) n_{\mathrm{e}}(r) T_{\mathrm{e}}(r) \frac{r \mathrm{~d} r}{\sqrt{r^{2}-R^{2}}}$

where the cluster is assumed to be spherically symmetric, $A_{\mathrm{SZ}}=$ $\sigma_{\mathrm{T}}\left(k T_{\mathrm{CMB}} / m_{\mathrm{e}} c^{2}\right), r$ is the physical radius from the cluster center, $R=D_{\mathrm{A}} \theta$ where $\theta$ is the projected angular distance on the sky and $D_{\mathrm{A}}$ is the angular diameter distance (to calculate $D_{\mathrm{A}}$, we assume the cosmology of Komatsu et al. 2009, for the remainder of the paper). $T_{\mathrm{e}}(r)$ and $n_{\mathrm{e}}(r)$ are the electron gas temperature and density radial profiles. We neglect the small $T_{\mathrm{e}}$ dependence in $f\left(x, T_{\mathrm{e}}\right)$ for this analysis and incorporate $f\left(x, T_{\mathrm{e}}=10 \mathrm{keV}\right)$ into the $A_{\mathrm{SZ}}$ factor.

For the de-projection analysis, the X-ray surface brightness profile can be written as (e.g. Yoshikawa \& Suto 1999):

$S_{\mathrm{X}}(R)=\frac{2}{4 \pi(1+z)^{4}} \int_{R}^{\infty} n_{\mathrm{e}}^{2}(r) \Lambda_{H}\left(T_{\mathrm{e}}(r)\right) \frac{r \mathrm{~d} r}{\sqrt{r^{2}-R^{2}}}$.

We use the APEC code (Smith et al. 2001) to compute the cooling function, $\Lambda\left(T_{\mathrm{e}}(r)\right)$ for Abell 2163, assuming an abundance value of $0.4 Z_{\odot}$, where $Z_{\odot}$ is the solar metallicity. This also includes the absorption by the neutral hydrogen of the Galaxy using a column density of $1.1 \times 10^{21} \mathrm{~cm}^{-2}$, as measured by the L.A.B. survey (Kalberla et al. 2005) at the cluster position. While the temperature dependence of the cooling function is thus included in the analysis, it is interesting to note that the results changes very little when assuming $\Lambda$ is independent of $T_{\mathrm{e}}$. As we shall see in Sect. 5.2, however, systematic effects in the gas density from uncertainties in the metal abundance are non-negligible.

Using Abel's integral equation, Eqs. (8) and (9) can be inverted to obtain joint radial density and temperature profiles:

$T_{\mathrm{e}}(r) n_{\mathrm{e}}(r)=\frac{1}{\pi A_{\mathrm{SZ}}} \int_{\infty}^{r} \frac{\mathrm{d} \Delta T(R)}{\mathrm{d} R} \frac{\mathrm{d} R}{\sqrt{R^{2}-r^{2}}} ;$
$n_{\mathrm{e}}^{2}(r) \Lambda\left(T_{\mathrm{e}}(r)\right)=4(1+z)^{4} \int_{\infty}^{r} \frac{\mathrm{d} S_{\mathrm{X}}(R)}{\mathrm{d} R} \frac{\mathrm{d} R}{\sqrt{R^{2}-r^{2}}}$.

Following Yoshikawa \& Suto (1999), we integrate Eqs. (10) and (11) numerically by summing in radial bins from $i_{\min }$ to $i_{\max }$, where $i_{\max }$ is the index for the outermost bin, and $i_{\min }$ corresponds to $r / D_{\mathrm{A}}$. We propagate the errors on the density and temperature profiles by a Monte-Carlo method, the details of which will be discussed in a future publication (Basu et al. 2009, in prep.).

\subsubsection{Profile extraction}

The SZE profile is derived as a radial average of the data and errors are estimated using the same method as in H09. In order to combine the two data sets, the X-ray raw mosaic has been smoothed to the APEX-SZ resolution of $1^{\prime}$. In doing so, we have neglected the effects of the XMM point spread function (PSF) and its variation over the field since they are negligible compared to the APEX-SZ beam. The X-ray source mask is used to remove the sub-cluster 8 arcmin north of Abell 2163, prominent in Fig. 3, and the numerous AGN. A count profile is then extracted from the background subtracted mosaic and corrected for the local exposure time and unmasked area, thus converting to surface brightness. The Poisson error bars on the counts profiles are re-scaled accordingly. Since the background and source counts in each annulus are large, the noise can be considered as Gaussian which justifies the direct background subtraction. Because of the slight offset between the peaks of the X-ray surface brightness and the SZE temperature decrement, we have taken the centroid position of the X-ray map to be the common center in the X-ray/SZE joint analysis because of the much better signal-to-noise ratio of the X-ray map.

\subsubsection{Mass estimation method}

Using the SZE and X-ray measurements, we can determine the gas mass and total mass enclosed within a certain radius assuming hydrostatic equilibrium. This can be used to compute the gas-to-mass ratio as a function of radial distance, or can be combined with the weak-lensing data for a more direct determination of the gas mass fraction. We show the comparison between the gas mass fraction values obtained using our non-parametric deprojection method and the standard isothermal modeling inside the $r_{500}$ of the cluster.

Computing the gas mass and total mass profiles from isothermal $\beta$ models is straightforward (e.g. LaRoque et al. 2006). The gas mass is obtained directly from the electron density profile as

$M_{\text {gas }}(<r)=4 \pi \mu_{\mathrm{e}} n_{\mathrm{e}}(0) m_{\mathrm{p}} D_{\mathrm{A}}^{3} \int_{0}^{r / D_{\mathrm{A}}}\left(1+\frac{\theta^{2}}{\theta_{\mathrm{c}}^{2}}\right)^{-3 \beta / 2} \theta^{2} \mathrm{~d} \theta$

where $\mu_{\mathrm{e}}$ is the mean molecular weight per electron, which we assume to be equal to 1.17 for cosmic abundance of $\mathrm{H}$ and $\mathrm{He}$. For the non-isothermal analysis, we use the de-projected electron density profile to compute the gas mass directly as $\rho_{\text {gas }}(r)=$ $\mu_{\mathrm{e}} m_{\mathrm{p}} n_{\mathrm{e}}(r)$.

The total mass, $M_{\text {total }}$, is obtained by solving the hydrostatic equilibrium (HSE) equation, assuming spherical symmetry, as follows:

$M_{\text {total }}(<r)=-\frac{k_{\mathrm{B}} T_{\mathrm{e}}(r) r}{G \mu m_{\mathrm{p}}}\left[\frac{\mathrm{d} \ln n_{\mathrm{e}}(r)}{\mathrm{d} \ln r}+\frac{\mathrm{d} \ln T_{\mathrm{e}}(r)}{\mathrm{d} \ln r}\right]$.

For isothermal modeling, we use the simple analytic form obtained from the above equation (e.g. Grego et al. 2001)

$M_{\text {total }}(<r)=\frac{3 \beta k_{\mathrm{B}} T_{\mathrm{e}}}{G \mu m_{\mathrm{p}}} \frac{r^{3}}{r^{2}+r_{\mathrm{c}}^{2}}$,

whereas for non-isothermal modeling we solve Eq. (13) directly. The gas mass fraction is in both cases computed as $f_{\text {gas }}(<r)=$ $M_{\text {gas }}(<r) / M_{\text {total }}(<r)$.

\subsection{Systematics}

The dominating source of error in the non-parametric deprojection analysis comes from uncertainties in the SZE map at $150 \mathrm{GHz}$. Intrinsic noise properties in the raw map cause the uncertainties in the radial bins to be strongly correlated due to noise structures much more extended than the bin width. In addition, the deconvolution process discussed in Sect. 3.3 not only recovers source signal, but can also amplify noise structures in 
the raw map, thus increasing the uncertainties in the radial profile (see Fig. 4). This amplification of noise effectively limits the range of radii for which we can make meaningful estimates of the ICM temperature and density.

As already discussed in Sect. 4.4.4, some modes corresponding to large angular scales are irrecoverable by the transfer function - in particular, no structures more extended than the path traced by a single bolometer during a scan can be recovered. Although the SZE profile is well constrained within $r_{500}$, this inherent filtering can lead to systematic shifts in temperature and density at larger radii, where the SZE signal is potentially underestimated.

Cavaliere et al. (2005) showed that the Abel deprojection method in itself can lead to biases in the estimated temperature and density profiles. However, they also found that this bias is significantly smaller than the statistical uncertainty, which they optimistically assumed to be $1 \%$ on the central value.

Other systematic uncertainties come from primary CMB signals, modeling of the X-ray background, and the metal abundance used in computing the X-ray cooling function. We consider each source of systematic uncertainty in what follows. The results are summarized in Table 2. Representative radial scales are taken from the best-fit NFW model from weak-lensing analysis of this cluster (Radovich et al. 2008): $r_{2500}=3.1^{\prime}$ and $r_{500}=7.6^{\prime}$.

\subsubsection{The filter function}

To address the issue of how irrecoverable signals on large angular scales in the SZE map affect the temperature and density profiles, we once again carry out a series of simulations (cf. Sect. 4.4.4), passing artificial models through the reduction pipeline. While in Sect. 4.4 we considered only how the signal was affected by the pipeline in terms of fitting a parametric function out to a certain radius using the transfer function, we now also have to consider systematic effects from using the transfer function to deconvolve the map rather than to convolve a model (the latter is much more straightforward). Lacking a more complete model of the cluster emission, we again use spherical $\beta$ models, and vary the best-fit parameters from Sect. 4 within their $1 \sigma$ errors to test for stability. After passing the models through the pipeline, we deconvolve them using the transfer function and compare the resulting profiles with the input models. While the systematic errors estimated from this method are comparable to the random errors on the SZE profile inside $r_{500}$, the signal is systematically lowered by as much as $40 \%$ of the input signal at $r_{200}$. Note, however, that at any radius considered in our analysis, this systematic effect is small compared to the intrinsic statistical error originating in the raw map.

It should be stressed that these error estimates are only meaningful in the context of the isothermal model. They are justified, however, by the results of Sect. 4, which indicate that this model indeed provides a very reasonable fit to the data out to $r_{500}$. In contrast to what was done in the isothermal analysis, we do not attempt to correct for this effect since we have no parametric model. Instead, we treat the systematic as an added uncertainty on the SZE profile, and propagate this uncertainty through the de-projection analysis to estimate the resulting uncertainties in temperature and density.

\subsubsection{Primary CMB}

The systematic uncertainty from the primary CMB signal is estimated using a method analogous to that described in Sect. 4.4.
Table 2. Systematic effects in the de-projected temperature and density profiles. Statistical errors are indicated for comparison.

\begin{tabular}{|c|c|c|c|c|}
\hline \multirow{2}{*}{$\begin{array}{l}\text { Source of } \\
\text { uncertainty }\end{array}$} & \multicolumn{2}{|c|}{ Effect on $T_{\mathrm{e}}$} & \multicolumn{2}{|c|}{ Effect on $n_{\mathrm{e}}$} \\
\hline & $r_{2500}$ & $r_{500}$ & $r_{2500}$ & $r_{500}$ \\
\hline Primary CMB & $\pm 3.7 \%$ & $\pm 8.7 \%$ & $\pm 1.1 \%$ & $\pm 3.3 \%$ \\
\hline Filter function (SZE map) & $+2.1 \%$ & $+8.9 \%$ & $+0.3 \%$ & $+1.4 \%$ \\
\hline X-ray background & $\begin{array}{l}+0.5 \% \\
-0.3 \%\end{array}$ & $\begin{array}{l}+2.4 \% \\
-3.1 \%\end{array}$ & ${ }_{-0.2 \%}^{+0.8 \%}$ & $\begin{array}{l}+1.1 \% \\
-0.3 \%\end{array}$ \\
\hline Metal abundance & $\begin{array}{l}+2.2 \% \\
-1.2 \%\end{array}$ & $\begin{array}{l}+1.5 \% \\
-0.2 \%\end{array}$ & $\begin{array}{l}+1.9 \% \\
-2.8 \%\end{array}$ & $\begin{array}{l}+2.4 \% \\
-3.5 \%\end{array}$ \\
\hline Total systematic ${ }^{a}$ & $\begin{array}{l}+4.8 \% \\
-3.9 \% \\
\end{array}$ & $\begin{array}{l}+12.8 \% \\
-9.2 \%\end{array}$ & $\begin{array}{l}+2.4 \% \\
-3.0 \%\end{array}$ & $\begin{array}{l}+4.5 \% \\
-4.8 \%\end{array}$ \\
\hline Statistical & $\pm 19.3 \%$ & $\pm 43.5 \%$ & $\pm 1.1 \%$ & $\pm 6.0 \%$ \\
\hline
\end{tabular}

${ }^{a}$ Added in quadrature.

After a set of simulated primary-CMB maps are subtracted from time stream data and passed through the pipeline reduction, deconvolution is performed on each map in the set. The different maps are then passed through the de-projection analysis to estimate the impact on the derived quantities. It is found that the uncertainties are comparable with those of the filter function.

\subsubsection{X-ray background and metal abundance}

Although the statistical errors on the recovered temperature and density profiles are clearly dominated by the uncertainty in the SZE signal, several X-ray systematics have to be considered.

As mentioned in Sect. 3.5, the background model can have a significant impact on the X-ray surface brightness at large radii. To quantify this further, we fix all the parameters of the background model to their best fit value, and allow for an overall change in normalization in the instrumental and cosmic background. Identifying the Cash statistic with a $\chi^{2}$ distribution (valid in the large number limit), we derive a $1 \sigma$ uncertainty for this normalization factor of $+1.9 \% /-1.2 \%$. At $r_{500}$, this translates into a $3 \%$ uncertainty in the temperature profile and $1 \%$ for the electron density, much below the statistical errors.

Our assumption of a fixed metal abundance is another potential source of systematics. Line emission is indeed preeminent in the soft energy band and this could result in a significantly different value of the cooling function required for our de-projection analysis. We have thus investigated the impact of a global change in metallicity in the range 0.2 to $0.8 Z_{\odot}$. These values correspond to the most extreme $1 \sigma$ bounds measured anywhere in the cluster by Snowden et al. (2008) from X-ray spectroscopy, yet they only change our results by up to few per cent, again smaller than the statistical uncertainties. The small systematic uncertainties are a direct consequence of the high temperatures measured for this cluster; line emission at soft energies is indeed much reduced for plasma hotter than $\sim 2 \mathrm{keV}$ in which more energetic transitions are favored.

\subsection{Results of the non-isothermal modeling}

\subsubsection{Density and temperature profiles}

The radial profiles of the ICM density and temperature, obtained from Eqs. (10) and (11), are shown in Fig. 5. The density profile shows very little deviation from an isothermal $\beta$ model inside $r_{500}$ (roughly $1500 \mathrm{kpc}$ ), which is expected since the density values are mostly constrained by the X-ray surface brightness 

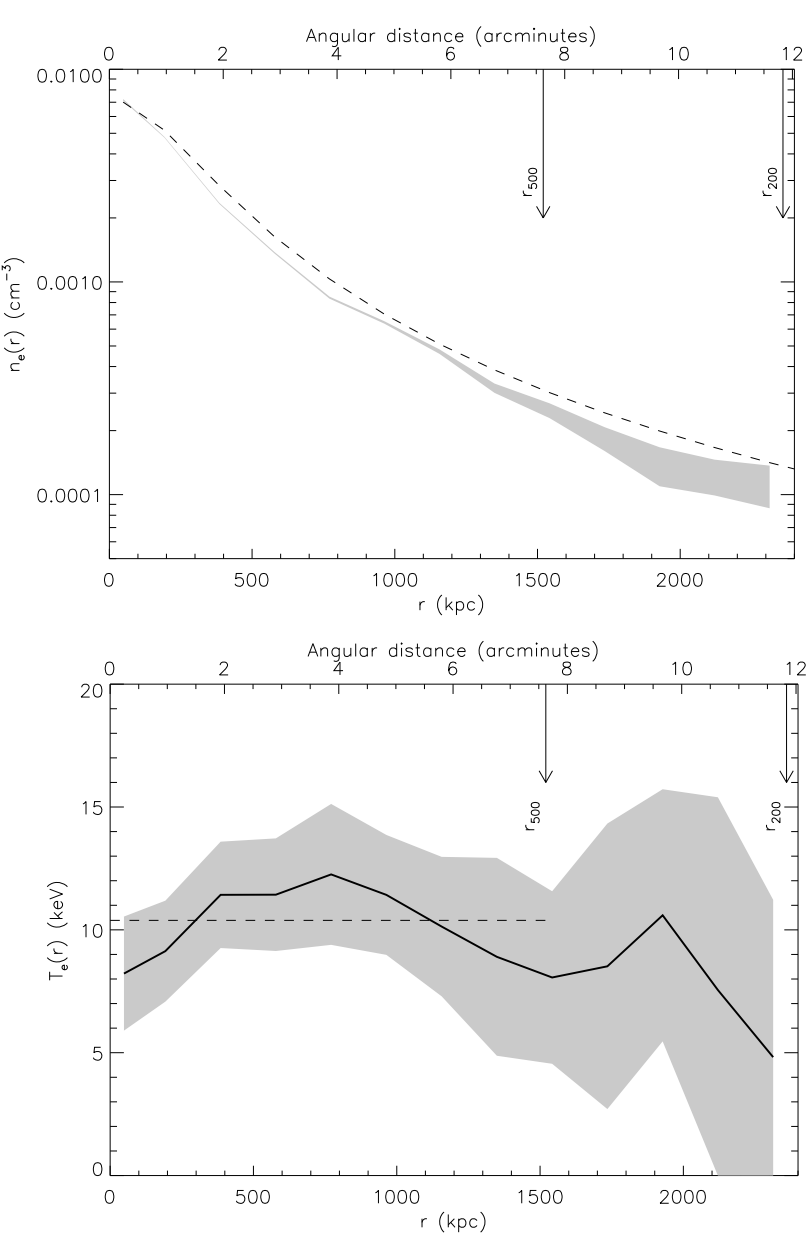

Fig. 5. De-projected radial density and temperature profiles of Abell 2163 from X-ray (XMM-Newton) and APEX-SZ data. Top: deprojected radial density profile ( $1 \sigma$ confidence region). The dashed line is the X-ray derived spherical $\beta$ profile $\left(\beta=0.64\right.$ and $\left.\theta_{\mathrm{c}}=91^{\prime \prime}\right)$, normalized to the derived central electron density value. Bottom: de-projected temperature profile with $1 \sigma$ uncertainties. The horizontal dashed line shows the best-fit isothermal value of $10.4 \pm 1.4 \mathrm{keV}$ within $r_{500}\left(\sim 7.6^{\prime}\right)$. Vertical arrows in both plots mark the estimated values of $r_{200}$ and $r_{500}$.

map. In the [0.5-2] keV energy band the X-ray surface brightness depends weakly on the gas temperature; for this reason the de-projected density profile using this band has a weak dependence on temperature variations. Inside $r_{500}$ the temperature can be fit with a constant (i.e. isothermal) value, at $10.4 \pm 1.4 \mathrm{keV}$, marked by the horizontal dashed line in Fig. 5.

Since no "de-projected" temperature profile for Abell 2163 from X-ray spectroscopic measurements is available in the literature, we compute the mean weighted value of the gas temperature along the line of sight to compare our results with the published X-ray temperature values. The projected gas temperature is computed as $T_{\text {proj }} \equiv \int W T \mathrm{~d} V / \int W \mathrm{~d} V$, where $T$ is the de-projected gas temperature obtained from Abel inversion, and $W$ is the weight function. As expected, the effect of projection is small compared to the errors in our temperature profile. We compare two different weighing schemes: the standard emission weight with $W=n^{2} \Lambda(T)$, and the weighing for a "spectroscopiclike" temperature as discussed by Mazzotta et al. (2004), using $W=n^{2} T^{-3 / 4}$. The difference between these two weighing schemes is negligible, as can be expected from the slowly varying temperature profile of A2163 which shows no strong nonisothermal features.

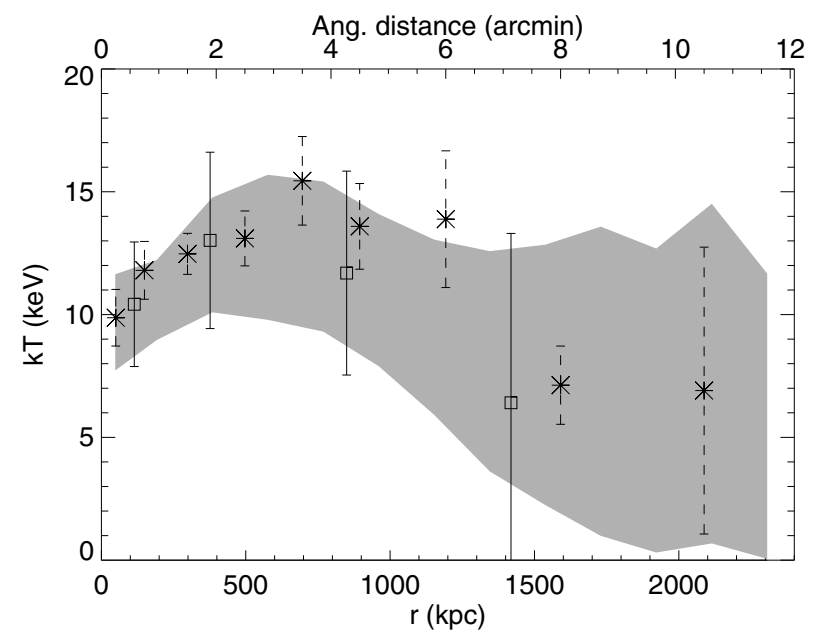

Fig. 6. Comparison of the projected radial temperature profile for Abell 2163 with spectroscopic X-ray measurements. The shaded region shows the emission-weighted temperature computed from the deprojected temperature and density profiles. The squares and crosses are X-ray spectroscopic measurements from Chandra (Markevitch \& Vikhlinin 2001) and XMM-Newton (Snowden et al. 2008), respectively.

In Fig. 6 we compare the emission-weighted temperature with published spectroscopic measurements from XMM-Newton and Chandra observations. The XMM-Newton temperature profile indicates a drop in temperature in the central region, which may be the remnant of the cold core of the original more massive cluster in this merging system. Gas temperatures derived from the APEX-SZ measurement are consistent with this feature. In the outskirts, gradually decreasing temperatures with incresing radii have been observed for relaxed clusters (e.g. Pratt et al. 2007), and is expected from both theory (e.g. Frenk et al. 1999) and numerical simulations of cluster models (e.g. Roncarelli et al. 2006; Hallman et al. 2007). Although the large uncertainties in the present temperature profile beyond $r_{500}$ do not allow any conclusions as to a decrease in temperature at large radii, the data are fully consistent with such a trend.

\subsubsection{Gas mass and total mass}

The results of the mass analysis are shown in Fig. 7. We have used median smoothing to the temperature and density profiles before computing their derivatives, and limited this analysis to within $r_{500}$ (approximately $1500 \mathrm{kpc}$ ) to avoid the large uncertainties at the outer radii. The errors in the total mass and gas mass fraction profiles reflect the combined random and systematic errors in our analysis. We find that the cumulative mass profile obtained under the assumption of HSE with spherical symmetry is in good agreement with the total mass obtained from weak lensing (Squires et al. 1997). The present analysis provides a better constraint on the total mass profile if the assumption of HSE is valid. The cumulative $f_{\text {gas }}$ values near the cluster center obtained from the non-parametric modeling are systematically higher than the isothermal $\beta$-model predictions. The drop to zero of the gas mass fraction at small radii is a well-known artefact of the isothermal $\beta$-modeling of cluster ICM, and our non-parametric profile may be indicative of the actual ratio between gas and total gravitational matter near the cluster center. At a radius of $1 \mathrm{Mpc}$ and beyond, the $f_{\text {gas }}$ values from both methods agree within $1 \sigma$. 

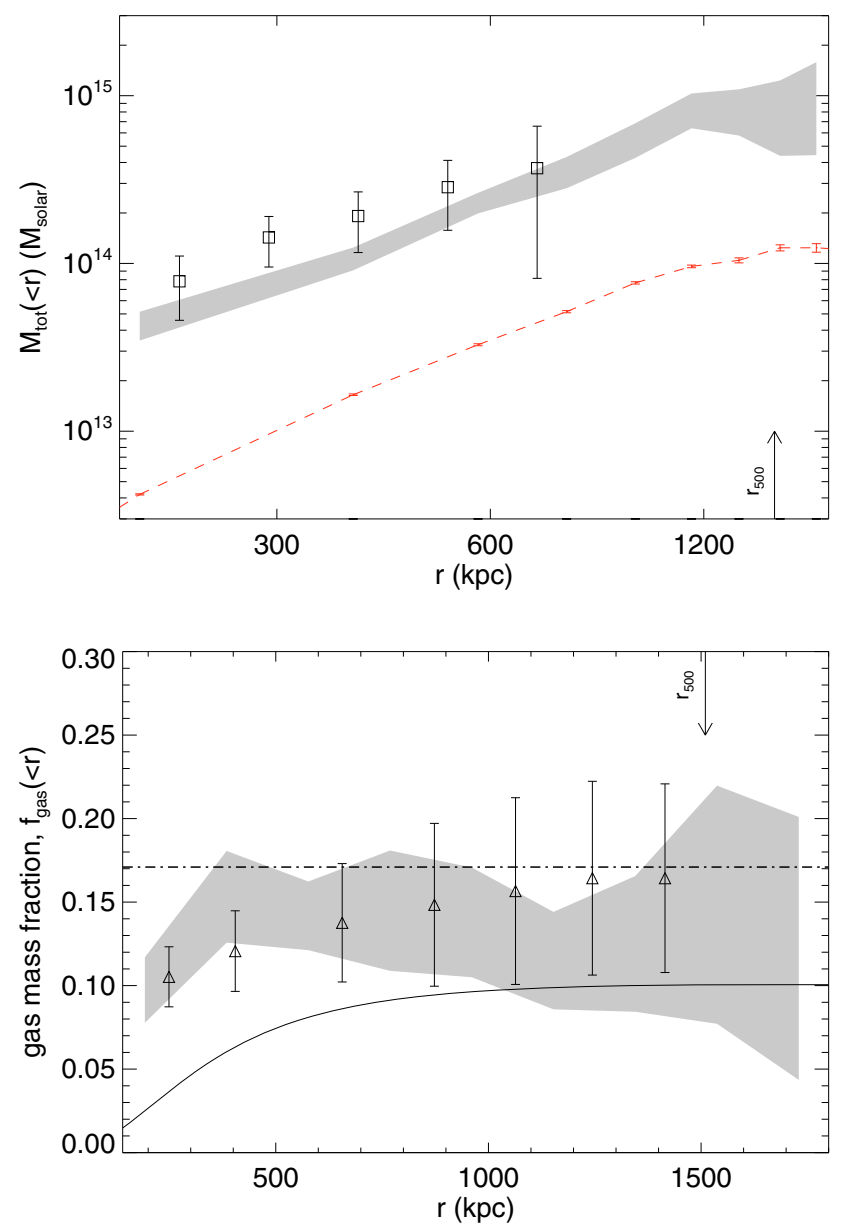

Fig. 7. Top: gas mass and total mass in Abell 2163 within $r_{500}$ $(\sim 1500 \mathrm{kpc})$, obtained from the de-projected density and temperature profiles. The shaded region shows the total mass within $68 \%$ confidence levels. The dashed line is the gas mass, constrained primarily from the X-ray data. The square boxes with error bars are the weaklensing mass profile from Squires et al. (1997). Bottom: the gas mass fraction obtained from the isothermal analysis (solid line) and from the non-parametric de-projection (shaded region, $68 \% \mathrm{CL}$ ). The triangles with error bars are the results from the X-ray analysis of Squires et al. (1997), and the horizontal dot-dashed line represents the cosmic baryon fraction from the WMAP 5-year result (Dunkley et al. 2009).

The resulting non-parametric gas mass fraction profile for Abell 2163 is close to the universal baryon fraction obtained from the WMAP 5-year data (Dunkley et al. 2009), and is also consistent with the X-ray analysis of Squires et al. (1997) for this cluster. The joint SZE/X-ray analysis of the gas fraction in clusters using OVRO/BIMA data (LaRoque et al. 2006) also yields a value of $f_{\text {gas }}$ in the range $0.15-0.16$ for this cluster, using a nonisothermal double $\beta$-model. The latter measurement is limited to within $r_{2500}$, and at this radius the SZE-only isothermal value is approximately $30 \%$ lower than the double $\beta$-model result, consistent with the difference found in our analysis.

It should be noted that the plotted mass and gas mass fractions are cumulative functions, and hence their values in the outer bins are correlated with the data in bins closer to the center. Furthermore, the errors in $f_{\text {gas }}$ are derived from the errors in the total mass and gas mass profiles, which are not independent. For these reasons, the errors in $f_{\text {gas }}$ are also correlated.

\section{Constraints from the SZE spectrum}

To compare the SZE decrement/increment values at different frequencies, we write them in terms of the relative change to the background CMB intensity,

$\Delta I=I_{0} h(x) \Omega_{\text {beam }} \frac{\Delta T}{T_{\mathrm{CMB}}}$,

where $x \equiv h v / k T_{\mathrm{CMB}}, I_{0} \equiv 2\left(k T_{\mathrm{CMB}}\right)^{3} /(h c)^{2}, \Omega_{\text {beam }}$ is the beam equivalent solid angle in steradians, and $h(x)$ relates the frequency dependence of the SZE expressed in temperature and in intensity as

$h(x)=\frac{x^{4} \mathrm{e}^{x}}{\left(\mathrm{e}^{x}-1\right)^{2}}$.

The total change of intensity is written as the sum of the thermal and kinematic components of the SZE as

$\Delta I=\Delta I_{\mathrm{T}}+\Delta I_{\mathrm{K}}$,

where

$\Delta I_{\mathrm{T}}=I_{0} y h(x) f\left(x, T_{\mathrm{e}}\right)$

and

$\Delta I_{\mathrm{K}}=-I_{0} y h(x)\left(m_{\mathrm{e}} c^{2} / k_{\mathrm{B}} T_{\mathrm{e}}\right) \frac{v_{r}}{c}$,

with $v_{r}$ the radial (line-of-sight) peculiar velocity, which is positive for a receding cluster.

Relativistic corrections to third order in $k_{\mathrm{B}} T_{\mathrm{e}} / m_{\mathrm{e}} c^{2}$ are included in $f\left(x, T_{\mathrm{e}}\right)$ according to Sazonov \& Sunyaev (1998) (Note that the quantity $h(x) f\left(x, T_{\mathrm{e}}\right)$ is often denoted $g\left(x, T_{\mathrm{e}}\right)$ in the literature). Higher order relativistic corrections are negligible given the precision of the current measurements. For the purpose of fitting the SZE spectrum, the Comptonization parameter $y$ (defined in Eq. (3) is parameterized in terms of its central value $y_{0}$. This allows a simple model where the finite resolutions of different experiments are accounted for. All the spectral data are obtained with a radial fit using spherical $\beta$ models, and accounting for beam dilution. For APEX-SZ and LABOCA, we use the central decrement/increment values, corrected for dust contamination, derived in Sect. 4. The data used for the fit to the SZE spectrum are given in Table 3.

Due to the degeneracies between velocity, temperature and Comptonization, the present data is not sufficient for a simultaneous constraint of all three parameters. Instead, we fix the ICM temperature to $10.4 \mathrm{keV}$ from the joint X-ray and SZE analysis of Sect. 5, and perform a least squares fit in $y_{0}$ and $v_{r}$. We also fit these parameters using alternative values of the temperature in the range $8-14 \mathrm{keV}$.

To estimate the errors in the fitted parameters, we perform a Monte Carlo simulation, in which 10000 artificial data sets are created from the actual spectral data, adding random Gaussian offsets with the amplitude of the statistical noise at each frequency. To model the effect of the systematics, we also add random offsets from the most important systematic components from Sect. 4.4, with the proper correlations between the different frequencies taken into account. Dust and primary CMB signals are scaled to the estimated levels derived in Sect. 4 at 150 and $345 \mathrm{GHz}$ and to the levels derived by LaRoque et al. (2002) at the other frequencies. To account for primary calibration uncertainties, we assume a 5\% calibration uncertainty across the spectrum. 

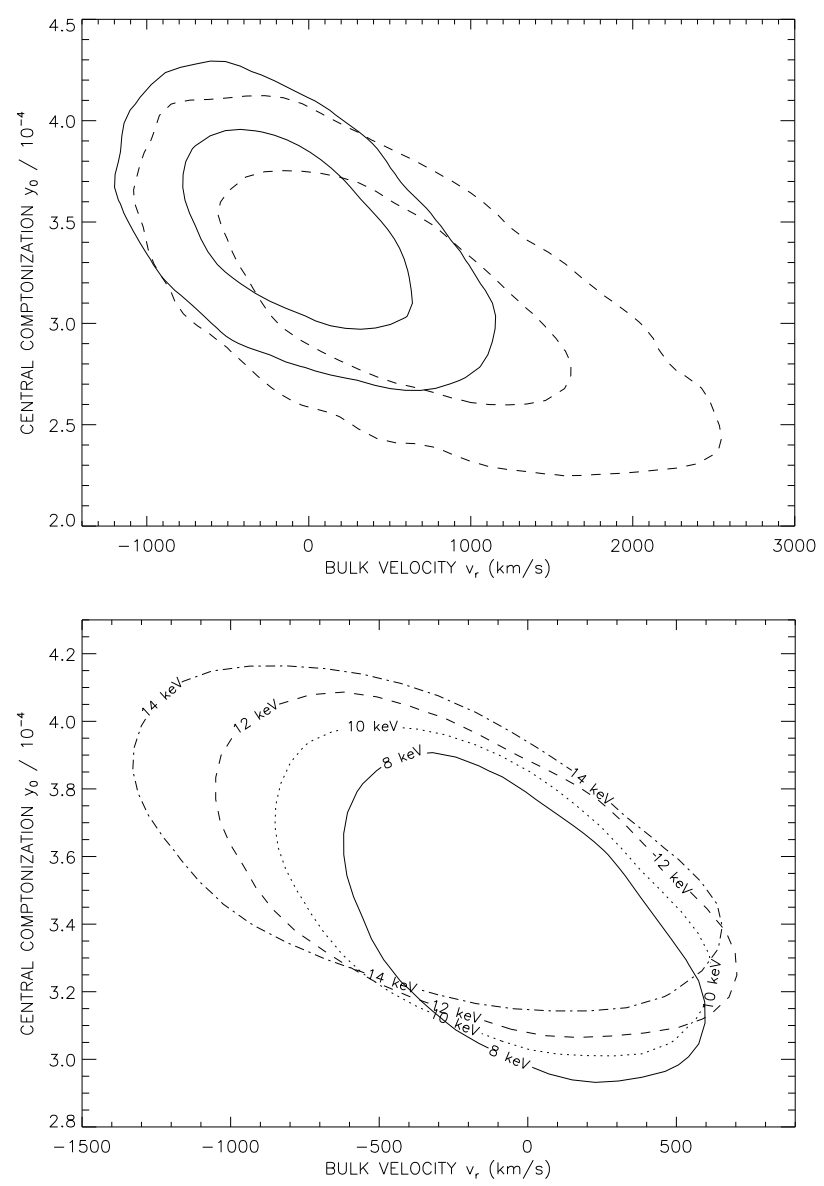

Fig. 8. Constraints in the $v_{r}-y_{0}$ parameter space of Abell 2163 with priors on the ICM temperature, including systematic uncertainties. Top: solid contours indicate the $68 \%$ and $95 \%$ confidence regions using all available data and assuming $k T_{\mathrm{e}}=10.4 \mathrm{keV}$. The dashed contours correspond to excluding the present (APEX-SZ and LABOCA) measurements. Bottom: $68 \%$ confidence regions for a range of ICM temperatures (indicated) using all available data.

Table 3. SZE decrement/increment measurements used for the spectral fit.

\begin{tabular}{llc}
\hline \hline Wavelength $(\mathrm{mm})$ & Instrument & $\Delta I\left(\mathrm{MJy} \mathrm{sr}^{-1}\right)$ \\
\hline 10 & OVRO/BIMA $^{1}$ & $-0.043 \pm 0.005^{4}$ \\
2.1 & SuZIE $^{2}$ & $-0.342 \pm 0.033^{3,4}$ \\
2.0 & APEX-SZ & $-0.317 \pm 0.035$ \\
1.4 & SuZIE $^{2}$ & $-0.093 \pm 0.069^{3,4}$ \\
1.1 & SuZIE $^{2}$ & $0.266 \pm 0.095^{3,4}$ \\
0.86 & LABOCA & $0.633 \pm 0.094$ \\
\hline
\end{tabular}

${ }^{1}$ LaRoque et al. (2002); ${ }^{2}$ Holzapfel et al. (1997); ${ }^{3}$ dust-corrected intensities from LaRoque et al. (2002); ${ }^{4}$ calibration corrected according to Hill et al. (2009).

The resulting error estimates, including systematics, are shown in Fig. 8. The fitted radial velocity of the cluster is consistent with zero, in agreement with and marginally improving the constraints of LaRoque et al. Specifically, we find $v_{r}=$ $-140 \pm 460 \mathrm{~km} \mathrm{~s}^{-1}$ and $y_{0}=3.42 \pm 0.32 \times 10^{-4}$, excluding systematic effects. The fit is dominated by random noise; we find that including the systematic errors in the modeling increases the error in $y_{0}$ by $12 \%$ and in $v_{r}$ by $18 \%$. The SZE spectra resulting from the various fits are indicated in Fig. 9.

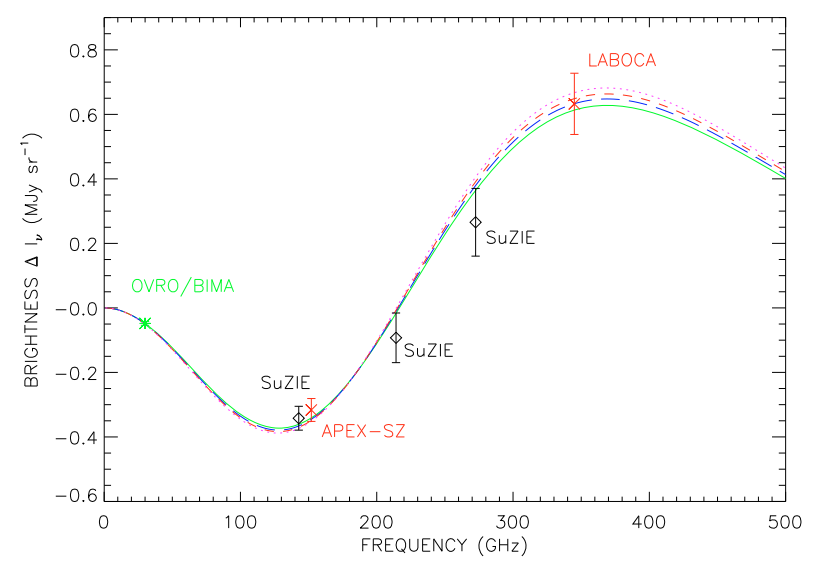

Fig. 9. SZE spectrum of Abell 2163 (points) and best-fit models using different priors on the ICM temperature: $8 \mathrm{keV}$ (solid line), $10 \mathrm{keV}$ (long-dashed line), $12 \mathrm{keV}$ (short-dashed line) and $14 \mathrm{keV}$ (dotted line).

\section{Conclusions}

1. We present SZE maps of the galaxy cluster Abell 2163 at two frequencies, showing the SZE decrement at $150 \mathrm{GHz}$ from observations with the APEX-SZ bolometer camera, and the SZE increment at $345 \mathrm{GHz}$ from observations with the LABOCA bolometer camera. The $345 \mathrm{GHz}$ measurement is the highest resolution SZE image for this cluster to date, and the first large-area $\left(>10^{\prime}\right)$ imaging of a galaxy cluster at submm wavelengths.

2. An isothermal modeling of the SZE yields results consistent with the X-ray derived isothermal model fits, implying that the large-scale properties of the cluster, under the assumption of spherical symmetry, are well represented by such models.

3. Using the APEX-SZ map in conjunction with XMM-Newton $\mathrm{X}$-ray data we derive the (de-projected) radial density and temperature profile of the ICM under the assumption of spherical symmetry. The projected gas temperature profile is found to be fully consistent with previous X-ray spectroscopic measurements of Markevitch \& Vikhlinin (2001) using Chandra data, and Snowden et al. (2008) using $X M M-N e w t o n$ data.

4. The total mass profile of the cluster is obtained under the assumption of hydrostatic equilibrium. The resulting profile agrees well with the weak-lensing mass profiles from previous works (Squires et al. 1997; Radovich et al. 2008), and extends the profile out to $r_{500}$.

5. The gas mass fraction of the ICM obtained from our nonparametric analysis are consistent with the previous X-ray mesurements (Squires et al. 1997) and SZE/X-ray joint analysis with double $\beta$-model (LaRoque et al. 2006). We do not see any significant trend for increasing ICM baryonic fraction from $r_{2500}$ to $r_{500}$ of the cluster.

6. Using isothermal fits to the LABOCA and APEX-SZ measurements, we constrain the line-of-sight peculiar velocity of the cluster and the central optical depth to inverse Compton scattering, using the temperature obtained from the de-projection analysis. We find a peculiar velocity $v_{r}=$ $-140 \pm 460 \mathrm{~km} \mathrm{~s}^{-1}$, consistent with zero, and a central Comptonization $y_{0}=3.42 \pm 0.32 \times 10^{-4}$.

Acknowledgements. We thank the APEX staff for their unfailing support during the APEX-SZ and LABOCA observations. We thank Wilhelm Altenhoff for fruitful discussions on the calibration of the millimeter data, and Fred Schuller for helping with the LABOCA data taking. This work has been partially supported by the DFG Priority Program 1177. M.N. acknowledges 
support for this research through a stipend from the International Max Planck Research School (IMPRS) for Radio and Infrared Astronomy at the Universities of Bonn and Cologne. F.P. acknowledges support from the DfG Transregio Programme TR33. N.W.H. acknowedges support from an Alfred P. Sloan Research Fellowship. APEX is a collaboration between the Max-Planck-Institut für Radioastronomie, the European Southern Observatory, and the Onsala Space Observatory. APEX-SZ is funded by the National Science Foundation under Grant No. AST-0138348. XMM-Newton is an ESA science mission with instruments and contributions directly funded by ESA Member States and the USA (NASA). The XMM-Newton project is supported in Germany by the Bundesministerium für Wirtschaft und Technologie/Deutsches Zentrum für Luft- und Raumfahrt (BMWI/DLR, FKZ 50 OX 0001), the Max-Planck Society and the Heidenhain-Stiftung.

\section{References}

Ameglio, S., Borgani, S., Pierpaoli, E., \& Dolag, K. 2007, MNRAS, 382, 397 Bertin, E., \& Arnouts, S. 1996, A\&AS, 117, 393

Birkinshaw, M. 1999, Phys. Rep., 310, 97

Cash, W. 1979, ApJ, 228, 939

Cavaliere, A., \& Fusco-Femiano, R. 1978, A\&A, 70, 677

Cavaliere, A., Lapi, A., \& Rephaeli, Y. 2005, ApJ, 634, 784

Condon, J. J., Cotton, W. D., Greisen, E. W., et al. 1998, AJ, 115, 1693

Cooray, A. R., Grego, L., Holzapfel, W. L., Joy, M., \& Carlstrom, J. E. 1998, ApJ, 115, 1388

De Filippis, E., Sereno, M., Bautz, M. W., \& Longo, G. 2005, ApJ, 625, 108

Dobbs, M., Halverson, N. W., Ade, P. A. R., et al. 2006, NewAR, 50, 960

Dunkley, J., Komatsu, E., Nolta, M. R., et al. 2009, ApJS, 180, 306

Elbaz, D., Arnaud, M., \& Boehringer, H. 1995, A\&A, 293, 337

Enoch, M. L., Young, K. E., Glenn, J., et al. 2006, ApJ, 638, 293

Feretti, L., Brunetti, G., Giovannini, G., Kassim, N., \& Setti, G. 2004, A\&A, 423, 111

Finkbeiner, D., Davis, M., \& Schlegel, D. 1999, ApJ, 524, 867

Frenk, C. S., White, S. D. M., Bode, P., et al. 1999, ApJ, 525, 554

Gorski, K. M., Hivon, E., \& Banday, A. J. 2005, ApJ, 622, 759

Govoni, F., Markevitch, M., Vikhlinin, A., et al. 2004, ApJ, 605, 695

Grego, L., Carlstrom, J. E., Reese, E. D., et al. 2001, ApJ, 552, 2

Griffin, M. J., \& Orton, G. S. 1993, Icar, 105, 537

Hallman, E. J., Burns, J. O., Motl, P. M., \& Norman, M. L. 2007, ApJ, 665, 911

Halverson, N., Lanting, T., Ade, P. A. R., et al. 2009, ApJ, 701, 42 (H09)

Hansen, S. H., Pastor, S., \& Semikoz, D. V. 2002, ApJ, 573, 169

Hill, R. S., Weiland, J. L., Odegard, N., et al. 2009, ApJS, 180, 246

Högbom, J. A. 1974, A\&AS, 15, 417

Holzapfel, W. L., Ade, P. A. R., Church, S. E., et al. 1997a, ApJ, 481, 35

Holzapfel, W. L., Arnaud, M., \& Ade, P. A. R. 1997b, ApJ, 480, 449
Kalberla, P. M. W., Burton, W. B., Hartmann, D., et al. 2005, A\&A, 440, 775

Kitayama, T., Komatsu, E., Ota, N., et al. 2004, PASJ, 56, 17

Komatsu, E., Dunkley, J., Nolta, M. R., et al. 2009, ApJS, 180, 330

Kosowsky, A. 2006, NewAR, 50, 969

Kreysa, E., Bertoldi, F., Gemuend, H.-P., et al. 2003, PIE, 4855, 41

Lamarre, J. M., Giard, M., Pointecouteau, E., et al. 1998, ApJ, 507, 5L

LaRoque, S. J., Carlstrom, J. E., Reese, E. D., et al. 2002

[arXiv: astro-ph/0204134]

LaRoque, S., Bonamente, M., Carlstrom, J. E., et al. 2006, ApJ, 652, 917

Lee, J., \& Suto, Y. 2004, ApJ, 601, 599L

Loeb, A., \& Refregier, A. 1997, ApJ, 476, 59L

Markevitch, M., \& Vikhlinin, A. 2001, ApJ, 563, 95

Mathiesen, B., Evrard, A. E., \& Mohr, J. 1999, ApJ, 520, L21

Maurogordato, S., Cappi, A., Ferrari, C., et al. 2008, A\&A, 481, 593

Mazzotta, P., Rasia, E., Moscardini, L., \& Tormen, G. 2004, MNRAS, 354, 10

Muhleman, D. O., \& Berge, G. L. 1991, Icarus, 92, 263

Nord, M. 2009, Ph.D. Thesis

Pratt, G. W., \& Arnaud, M. 2002, A\&A, 394, 375

Pratt, G. W., Böhringer, H., Croston, J. H., et al. 2007, A\&A, 461, 71

Puchwein, E., \& Bartelmann, M. 2006, A\&A, 442, 405

Radovich, M., Puddu, E., Romano, A., Grado, A., \& Getman, F. 2008, A\&A, 487, 55

Reddy, N. A., \& Yun, M. S. 2004, ApJ, 600, 695

Reese, E. D., Carlstrom, J. E., Joy, M., et al. 2002, ApJ, 581, 53

Roncarrelli, M., Ettori, S., Dolag, K., et al. 2006, MNRAS, 373, 1339

Rudy, D. J., Muhleman, D. O., Berge, G. L., et al. 1987, Icarus, 71, 159

Ruhl, J. E., Ade, P. A. R., Carlstrom, J. E., et al. 2004, SPIE, 5498, 11

Sazonov, S., \& Sunyaev, R. 1998, ApJ, 508, 1

Schlegel, D., Finkbeiner, D., \& Davis, M. 1998, ApJ, 500, 525

Schwan, D., Bertoldi, F., Cho, S., et al. 2003, NewAR, 47, 933

Schwarz, U. J. 1978, A\&A, 65, 345

Silk, J., \& White, S. 1978, ApJ, 226L

Siringo, G., et al. 2009, A\&A, submitted

Smith, R., Brickhouse, N. S., Liedahl, D. A., \& Raymond, J. C. 2001, ApJ, 556, 91

Squires, G., Neumann, D., Kaiser, N., et al. 1997, ApJ, 482, 648

Staniszewski, Z., Ade, P. A. R., Aird, K. A., et al. 2009, ApJ, 701, 32

Sunyaev, R. A., \& Zel'dovich, Y. B. 1970, Ap\&SS, 7, 3

Ulich, B. L. 1981, AJ, 86, 1619

Weiss, A., Kovacs, A., Güsten, R., et al. 2008, A\&A, 490, 77

White, M., \& Majumdar, S. 2004, ApJ, 602, 565

Wright, E. L. 1976, ApJ, 210, 250

Yoshikawa, K., \& Suto, Y. 1999, ApJ, 513, 549

Zaldarriaga, M., \& Seljak, U. 2000, ApJS, 129, 431

Zaroubi, S., Squires, G., de Gasperis, G., et al. 2001, ApJ, 561, 600 